\title{
On pattern-avoiding partitions
}

\author{
Vít Jelínek* \\ Department of Applied Mathematics, Charles University, Prague \\ jelinek@kam.mff.cuni.cz \\ Toufik Mansour \\ Department of Mathematics, Haifa University, 31905 Haifa, Israel \\ toufik@math.haifa.ac.il \\ Submitted: Apr 17, 2007; Accepted: Mar 5, 2008; Published: Mar 12, 2008 \\ Mathematics Subject Classification: Primary 05A18; Secondary 05E10, 05A15, 05A17, 05A19
}

\begin{abstract}
A set partition of size $n$ is a collection of disjoint blocks $B_{1}, B_{2}, \ldots, B_{d}$ whose union is the set $[n]=\{1,2, \ldots, n\}$. We choose the ordering of the blocks so that they satisfy $\min B_{1}<\min B_{2}<\cdots<\min B_{d}$. We represent such a set partition by a canonical sequence $\pi_{1}, \pi_{2}, \ldots, \pi_{n}$, with $\pi_{i}=j$ if $i \in B_{j}$. We say that a partition $\pi$ contains a partition $\sigma$ if the canonical sequence of $\pi$ contains a subsequence that is order-isomorphic to the canonical sequence of $\sigma$. Two partitions $\sigma$ and $\sigma^{\prime}$ are equivalent, if there is a size-preserving bijection between $\sigma$-avoiding and $\sigma^{\prime}$-avoiding partitions.

We determine all the equivalence classes of partitions of size at most 7 . This extends previous work of Sagan, who described the equivalence classes of partitions of size at most 3 .

Our classification is largely based on several new infinite families of pairs of equivalent patterns. For instance, we prove that there is a bijection between $k$ noncrossing and $k$-nonnesting partitions, with a notion of crossing and nesting based on the canonical sequence. Our results also yield new combinatorial interpretations of the Catalan numbers and the Stirling numbers.
\end{abstract}

\section{Introduction}

A partition of size $n$ is a collection $B_{1}, B_{2}, \ldots, B_{d}$ of nonempty disjoint sets, called blocks, whose union is the set $[n]=\{1,2, \ldots, n\}$. We will assume that $B_{1}, B_{2}, \ldots, B_{d}$ are listed

\footnotetext{
*Supported by the project MSM0021620838 of the Czech Ministry of Education, and by the grant GD201/05/H014 of the Czech Science Foundation.
} 
in increasing order of their minimum elements, that is, $\min B_{1}<\min B_{2}<\cdots<\min B_{d}$. In this paper, we will represent a partition of size $n$ by its canonical sequence, which is an integer sequence $\pi=\pi_{1} \pi_{2} \cdots \pi_{n}$ such that $\pi_{i}=k$ if and only if $i \in B_{k}$. For instance, 1231242 is the canonical sequence of the partition of $\{1,2, \ldots, 7\}$ with the four blocks $\{1,4\},\{2,5,7\},\{3\}$ and $\{6\}$.

Note that a sequence $\pi$ over the alphabet $[d]$ represents a partition with $d$ blocks if and only if it has the following properties.

- Each number from the set $[d]$ appears at least once in $\pi$.

- For each $i, j$ such that $1 \leq i<j \leq d$, the first occurrence of $i$ precedes the first occurrence of $j$.

We remark that sequences satisfying these properties are also known as restricted growth functions, and they are often encountered in the study of set partitions [21, 26] as well as other related topics, such as Davenport-Schinzel sequences [6, 13, 14, 19].

Throughout this paper, we identify a set partition with the corresponding canonical sequence, and we use this representation to define the notion of pattern avoidance among set partitions. Let $\pi=\pi_{1} \pi_{2} \cdots \pi_{n}$ and $\sigma=\sigma_{1} \sigma_{2} \cdots \sigma_{m}$ be two partitions represented by their canonical sequences. We say that $\pi$ contains $\sigma$, if $\pi$ has a subsequence that is order-isomorphic to $\sigma$; in other words, $\pi$ has a subsequence $\pi_{f(1)}, \pi_{f(2)}, \ldots, \pi_{f(m)}$, where $1 \leq f(1)<f(2)<\cdots<f(m) \leq n$, and for each $i, j \in[m], \pi_{f(i)}<\pi_{f(j)}$ if and only if $\sigma_{i}<\sigma_{j}$. If $\pi$ does not contain $\sigma$, we say that $\pi$ avoids $\sigma$. Our aim is to study the set of all the partitions of $[n]$ that avoid a fixed partition $\sigma$. In such context, $\sigma$ is usually called a pattern.

Let $P(n)$ denote the set of all the partitions of $[n]$, let $P(n ; \sigma)$ denote the set of all partitions of $[n]$ that avoid $\sigma$, and let $p(n)$ and $p(n ; \sigma)$ denote the cardinality of $P(n)$ and $P(n ; \sigma)$, respectively. We say that two partitions $\sigma$ and $\sigma^{\prime}$ are equivalent, denoted by $\sigma \sim \sigma^{\prime}$, if $p(n ; \sigma)=p\left(n ; \sigma^{\prime}\right)$ for each $n$.

The concept of pattern-avoidance described above has been introduced by Sagan [21], who considered, among other topics, the enumeration of partitions avoiding patterns of size three. In our paper, we extend this study to larger patterns. We give new criteria for proving the equivalence of partition patterns. By computer enumeration, we verify that our criteria describe all the equivalence classes of patterns of size $n \leq 7$.

Most of our results are applicable to patterns of arbitrary length. Some of these results may be of independent interest. For instance, let us define $k$-noncrossing and $k$-nonnesting partitions as the partitions that avoid the pattern $12 \cdots k 12 \cdots k$ and $12 \cdots k k(k-1) \cdots 1$, respectively. We will show that these two patterns are equivalent for every $k$, by constructing a bijection between $k$-noncrossing and $k$-nonnesting partitions. It is noteworthy, that a different concept of crossings and nestings in partitions has been considered by Chen et al. [3, 4], and this different notion of crossings and nestings also admits a bijection between $k$-noncrossing and $k$-nonnesting partitions, as has been shown in [4]. There is, in fact, yet another notion of crossings and nestings in partitions that has been studied by Klazar [13, 14]. 
Several of our results are proved using a correspondence between partitions and 0-1 fillings of polyomino shapes. This correspondence allows us to translate recent results on fillings of Ferrers shapes [6, 15] and stack polyominoes [20] into the terminology of pattern-avoiding partitions. The correspondence between fillings of shapes and patternavoiding partitions works in the opposite way as well: some of our theorems, proved in the context of partitions, imply new results about pattern-avoiding fillings of Ferrers shapes and pattern-avoiding ordered graphs.

Apart from these results, we also present a class of patterns equivalent to the pattern $12 \cdots k$. Notice that the partitions avoiding $12 \cdots k$ are precisely the partitions with fewer than $k$ blocks. The number of such partitions can be expressed as a sum of the Stirling numbers of the second kind. Thus, our result can be viewed as a new combinatorial interpretation of the Stirling numbers of the second kind. Similarly, by providing patterns equivalent to 1212, we provide a new combinatorial interpretation of the Catalan numbers.

In Section 2, we present basic facts about pattern-avoiding partitions, and we summarize previously known results. Our main results are collected in Section 3, where we present several infinite families of classes of equivalent patterns. In Sections 4-7, we present a systematic classification of patterns of size $n=4, \ldots, 7$. The classification is mostly based on the general results from Section 3, except for two isolated cases that need to be handled separately. In particular, in Section 4, we prove that the pattern 1123 is equivalent to the pattern 1212, thus completing the characterization of the patterns of size four and obtaining another new interpretation for the Catalan numbers. In Section 5, we prove the equivalence $12112 \sim 12212$, and explain its implications for the theory of pattern-avoiding ordered graphs and polyomino fillings.

\section{Basic facts and previous results}

Let us first establish some notational conventions that will be applied throughout this paper. For a finite sequence $S=s_{1} s_{2} \cdots s_{p}$ and an integer $k$, we let $S+k$ denote the sequence $\left(s_{1}+k\right)\left(s_{2}+k\right) \cdots\left(s_{p}+k\right)$. For a symbol $k$ and an integer $d$, the constant sequence $(k, k, \ldots, k)$ of length $d$ is denoted by $k^{d}$. To prevent confusion, we will use capital letters $S, T, \ldots$ to denote arbitrary sequences of positive integers, and we will use lowercase greek symbols $(\pi, \sigma, \tau, \ldots)$ to denote canonical sequences representing partitions.

An infinite sequence $a_{0}, a_{1}, \ldots$ is often conveniently represented by its exponential generating function (or EGF for short), which is the formal power series $F(x)=\sum_{n \geq 0} \frac{a_{n} x^{n}}{n !}$. We mostly deal with the generating functions of the sequences of the form $(p(n ; \pi))_{n \geq 0}$, where $\pi$ is a given pattern. We simply call such a generating function the EGF of the pattern $\pi$.

Let us summarize previous results relevant to our topic. Let $\exp (x)=\sum_{n \geq 0} \frac{x^{n}}{n !}$ and $\exp _{<k}(x)=\sum_{n=0}^{k-1} \frac{x^{n}}{n !}$. We first state two simple propositions, which already appear in $[21]$.

Proposition 1. A partition avoids the pattern $1^{k}$ if and only if each of its blocks has size 
less than $k$. The EGF of the pattern $1^{k}$ is equal to

$$
\exp \left(\exp _{<k}(x)-1\right)
$$

Proposition 2. A partition avoids the pattern $12 \cdots k$ if and only if it has fewer than $k$ blocks. The corresponding EGF is equal to

$$
\exp _{<k}(\exp (x)-1)
$$

We omit the proofs of these two propositions. Let us just remark that the formulas given above are obtained by standard manipulation of EGFs. A common generalization of these formulas can be found, e.g., in [9, Proposition II.2].

The enumeration of partitions with fewer than $k$ blocks is closely related to the Stirling numbers of the second kind $S(n, m)$, defined as the number of partitions of $[n]$ with exactly $m$ blocks (see sequence A008277 in [22]).

Sagan [21] has described and enumerated the pattern-avoiding classes $P(n ; \pi)$ for the five patterns $\pi$ of length three. We summarize the relevant results in Table 1. We again omit the proofs.

\begin{tabular}{l|l}
$\tau$ & $p(n ; \tau)$ \\
\hline \hline 111 & sequence A000085 in [22] \\
$112,121,122,123$ & $2^{n-1}$
\end{tabular}

Table 1: Number of partitions in $P(n ; \tau)$, where $\tau \in P(3)$.

\section{General classes of equivalent patterns}

In this section, we introduce the tools that will be useful in our study of pattern-avoidance, and we prove our key results. We begin by introducing a general relationship between pattern-avoidance in partitions and pattern-avoidance in fillings of restricted shapes. This approach will provide a useful tool for dealing with many pattern problems.

\subsection{Pattern-avoiding fillings of diagrams}

We will use the term diagram to refer to any finite set of the cells of the two-dimensional square grid. To fill a diagram means to write a non-negative integer into each cell.

We will number the rows of diagrams from bottom to top, so the "first row" of a diagram is its bottom row, and we will number the columns from left to right. We will apply the same convention to matrices and to fillings. We always assume that each row and each column of a diagram is nonempty. Thus, for example, when we refer to a diagram with $r$ rows, it is assumed that each of the $r$ rows contains at least one cell of the diagram. Note that there is a (unique) empty diagram with no rows and no columns. Let $r(F)$ and 
$c(F)$ denote, respectively, the number of rows and columns of $F$, where $F$ is a diagram, or a matrix, or a filling of a diagram.

We will mostly use diagrams of a special shape, namely Ferrers diagrams and stack polyominoes. We begin by giving the necessary definitions.

Definition 3. A Ferrers diagram, also called Ferrers shape, is a diagram whose cells are arranged into contiguous rows and columns satisfying the following rules.

- The length of any row is greater than or equal to the length of any row above it.

- The rows are right-justified, i.e., the rightmost cells of the rows appear in the same column.

We admit that our convention of drawing Ferrers diagrams as right-justified rather than left-justified shapes is different from standard practice; however, our definition will be more intuitive in the context of our applications.

Definition 4. A stack polyomino $\Pi$ is a collection of finitely many cells of the twodimensional rectangular grid, arranged into contiguous rows and columns with the property that for any $i=1, \ldots, r(\Pi)$, every column intersecting the $i$-th row also intersects all the rows with index smaller than $i$.

Clearly, every Ferrers shape is also a stack polyomino. On the other hand, a stack polyomino can be regarded as a union of a Ferrers shape and a vertically reflected copy of another Ferrers shape.

Definition 5. A filling of a diagram is an assignment of non-negative integers to the cells of the diagram. A $0-1$ filling is a filling that only uses values 0 and 1 . In such filling, a 0 -cell of a filling is a cell that is filled with value 0 , and a 1 -cell is filled with value 1 . A 0-1 filling is called semi-standard if each of its columns contains exactly one 1-cell. A 0-1 filling is called sparse if every column has at most one 1-cell. A column of a $0-1$ filling is called zero column if it contains no 1-cell. A zero row is defined analogously.

Among several possibilities to define pattern-avoidance in fillings, the following approach seems to be the most useful and most common.

Definition 6. Let $M=\left(m_{i j} ; i \in[r], j \in[c]\right)$ be a matrix with $r$ rows and $c$ columns with all entries equal to 0 or 1 , and let $F$ be a filling of a diagram. We say that $F$ contains $M$ if $F$ contains $r$ distinct rows $i_{1}<\cdots<i_{r}$ and $c$ distinct columns $j_{1}<\cdots<j_{c}$ with the following two properties.

- Each of the rows $i_{1}, \ldots, i_{r}$ intersects all columns $j_{1}, \ldots, j_{c}$ in a cell that belongs to the underlying diagram of $F$.

- If $m_{k \ell}=1$ for some $k$ and $\ell$, then the cell of $F$ in row $i_{k}$ and column $j_{\ell}$ has a nonzero value. 
If $F$ does not contain $M$, we say that $F$ avoids $M$. We will say that two matrices $M$ and $M^{\prime}$ are Ferrers-equivalent (denoted by $M \stackrel{\mathrm{F}}{\sim} M^{\prime}$ ) if for every Ferrers shape $\Delta$, the number of semi-standard fillings of $\Delta$ that avoid $M$ is equal to the number of semi-standard fillings of $\Delta$ that avoid $M^{\prime}$. We will say that $M$ and $M^{\prime}$ are stack-equivalent (denoted by $M \stackrel{\mathrm{s}}{\sim} M^{\prime}$ ) if the equality holds even for semi-standard fillings of an arbitrary stack polyomino.

Pattern-avoidance in the fillings of diagrams has received considerable attention lately. Apart from semi-standard fillings, various authors have considered standard fillings with exactly one 1-cell in each row and each column (see [2] or [23]), as well as general fillings with non-negative integers (see [7] or [15]). Also, nontrivial results were obtained for fillings of more general shapes (e.g. moon polyominoes [20]). These results often consider the cases when the forbidden pattern $M$ is the identity matrix (i.e., the $r \times r$ matrix, $I_{r}$, with $m_{i j}=1$ if and only if $i=j$ ) or the anti-identity matrix (i.e. the $r \times r$ matrix, $J_{r}$, with $m_{i j}=1$ if and only if $i+j=r+1$ ).

Since our next arguments mostly deal with semi-standard fillings, we will drop the adjective 'semi-standard' and simply use the term 'filling', when there is no risk of ambiguity.

Remark 7. Let $M$ and $M^{\prime}$ be two Ferrers-equivalent 0-1 matrices with a 1-cell in every column, and let $f$ be a bijection between $M$-avoiding and $M^{\prime}$-avoiding semi-standard fillings of Ferrers shapes. There is a natural way to extend $f$ into a bijection between $M$-avoiding and $M^{\prime}$-avoiding sparse fillings of Ferrers shapes. Assume that $F$ is a sparse $M$-avoiding filling of a Ferrers shape $\Delta$. The non-zero columns of $F$ form a semi-standard filling of a (not necessarily contiguous) subdiagram of $\Delta$. We apply $f$ to this subfilling to transform $F$ into a sparse $M^{\prime}$-avoiding filling of $\Delta$.

A completely analogous argument can be made for stack polyominoes instead of Ferrers shapes.

We now introduce some more notation, which will be useful for translating the language of partitions to the language of fillings.

Definition 8. Let $S=s_{1} s_{2} \cdots s_{m}$ be a sequence of positive integers, and let $k \geq$ $\max \left\{s_{i}: i \in[m]\right\}$ be an integer. We let $M(S, k)$ denote the 0-1 matrix with $k$ rows and $m$ columns which has a 1-cell in row $i$ and column $j$ if and only if $s_{j}=i$.

We now describe the correspondence between partitions and fillings of Ferrers diagrams (recall that $\tau+k$ denotes the sequence obtained from $\tau$ by adding $k$ to every element).

Lemma 9. Let $S$ and $S^{\prime}$ be two nonempty sequences over the alphabet $[k]$, let $\tau$ be an arbitrary partition. If $M(S, k)$ is Ferrers-equivalent to $M\left(S^{\prime}, k\right)$ then the partition pattern $\sigma=12 \cdots k(\tau+k) S$ is equivalent to $\sigma^{\prime}=12 \cdots k(\tau+k) S^{\prime}$.

Proof. Let $\pi$ be a partition of $[n]$ with $m$ blocks. Let $M$ denote the matrix $M(\pi, m)$. Fix a partition $\tau$ with $t$ blocks, and let $T$ denote the matrix $M(\tau, t)$. We will color the cells of $M$ red and green. If $\tau$ is nonempty, then the cell in row $i$ and column $j$ is colored green if 
and only if the submatrix of $M$ induced by the rows $i+1, \ldots, m$ and columns $1, \ldots, j-1$ contains $T$. If $\tau$ is empty, then the cell in row $i$ and column $j$ is green if and only if row $i$ has at least one 1-cell strictly to the left of column $j$. A cell is red if it is not green.

Note that the green cells form a Ferrers diagram, and the entries of the matrix $M$ form a sparse filling $G$ of this diagram. Also, note that the leftmost 1-cell of each row is always red, and any 0-cell of the same row to the left of the leftmost 1-cell is red too.

It is not difficult to see that the partition $\pi$ avoids $\sigma$ if and only if the filling $G$ of the 'green' diagram avoids $M(S, k)$, and $\pi$ avoids $\sigma^{\prime}$ if and only if $G$ avoids $M\left(S^{\prime}, k\right)$. Since $M(S, k) \stackrel{\mathrm{F}}{\sim} M\left(S^{\prime}, k\right)$, there is a bijection $f$ that maps $M(S, k)$-avoiding fillings of Ferrers shapes onto $M\left(S^{\prime}, k\right)$-avoiding fillings of the same shape. By Remark $7, f$ can be extended to sparse fillings. Using this extension of $f$, we construct the following bijection between $P(n ; \sigma)$ and $P\left(n ; \sigma^{\prime}\right)$ : for a partition $\pi \in P(n ; \sigma)$ with $m$ blocks, we take $M$ and $G$ as above. By assumption, $G$ is $M(S, k)$-avoiding. Using the bijection $f$ and Remark 7 , we transform $G$ into an $M\left(S^{\prime}, k\right)$-avoiding sparse filling $f(G)=G^{\prime}$, while the filling of the red cells of $M$ remains the same. We thus obtain a new matrix $M^{\prime}$.

Note that if we color the cells of $M^{\prime}$ red and green using the criterion described in the first paragraph of this proof, then each cell of $M^{\prime}$ will receive the same color as the corresponding cell of $M$, even though the occurrences of $T$ in $M^{\prime}$ need not correspond exactly to the occurrences of $T$ in $M$. Indeed, if $\tau$ is nonempty, then for each green cell $g$ of $M$, there is an occurrence of $T$ to the left and above $g$ consisting entirely of red cells. This occurrence is contained in $M^{\prime}$ as well, which guarantees that the cell $g$ remains green in $M^{\prime}$. A similar argument can be made if $\tau$ is empty.

By construction, $M^{\prime}$ has exactly one 1-cell in each column, hence there is a sequence $\pi^{\prime}$ over the alphabet $[m]$ such that $M^{\prime}=M\left(\pi^{\prime}, m\right)$. We claim that $\pi^{\prime}$ is a canonical sequence of a partition. To see this, note that for every $i \in[\mathrm{m}]$, the leftmost 1-cell of $M$ in row $i$ is red and the preceding 0 -cells in row $i$ are red too. It follows that the leftmost 1-cell of row $i$ in $M$ is also the leftmost 1-cell of row $i$ in $M^{\prime}$. Thus, the first occurrence of the symbol $i$ in $\pi$ appears at the same place as the first occurrence of $i$ in $\pi^{\prime}$, hence $\pi^{\prime}$ is indeed a partition. The green cells of $M^{\prime}$ avoid $M\left(S^{\prime}, k\right)$, so $\pi^{\prime}$ avoids $\sigma^{\prime}$. Obviously, the transform $\pi \mapsto \pi^{\prime}$ is invertible and provides a bijection between $P(n ; \sigma)$ and $P\left(n ; \sigma^{\prime}\right)$.

In general, the relation $12 \ldots k S \sim 12 \ldots k S^{\prime}$ does not imply that $M(S, k)$ and $M\left(S^{\prime}, k\right)$ are Ferrers equivalent. In Section 5, we will prove that $12112 \sim 12212$, even though $M(112,2)$ is not Ferrers equivalent to $M(212,2)$.

On the other hand, the relation $12 \ldots k S \sim 12 \ldots k S^{\prime}$ allows us to establish a somewhat weaker equivalence between pattern-avoiding fillings, using the following lemma.

Lemma 10. Let $S$ be a nonempty sequence over the alphabet $[k]$, and let $\tau=12 \cdots k S$. For every $n$ and $m$, there is a bijection $f$ that maps the set of $\tau$-avoiding partitions of $[n]$ with $m$ blocks onto the set of all the $M(S, k)$-avoiding fillings $F$ of Ferrers shapes that satisfy $c(F)=n-m$ and $r(F) \leq m$.

Proof. Let $\pi$ be a $\tau$-avoiding partition of $[n]$ with $m$ blocks. Let $M=M(\pi, m)$, and let us consider the same red and green coloring of $M$ as in the proof of Lemma 9, i.e., the 
green cells of a row $i$ are precisely the cells that are strictly to the right of the leftmost 1-cell in row $i$.

Note that $M$ has exactly $m$ red 1-cells, and each 1-cell is red if and only if it is the leftmost 1-cell of its row. Note also that if $c_{i}$ is the column containing the red 1-cell in row $i$, then either $c_{i}$ is the rightmost column of $M$, or column $c_{i}+1$ is the leftmost column of $M$ with exactly $i$ green cells.

Let $G$ be the filling formed by the green cells. As was pointed out in the previous proof, the filling $G$ is a sparse $M(S, k)$-avoiding filling of a Ferrers shape. Note that for each $i=1, \ldots m-1$, the filling $G$ has exactly one zero column of height $i$, and this column, which corresponds to $c_{i+1}$, is the rightmost of all the columns of $G$ with height at most $i$.

Let $G^{-}$be the subfilling of $G$ induced by all the nonzero columns of $G$. Observe that $G^{-}$is a semi-standard $M(S, k)$-avoiding filling of a Ferrers shape with exactly $n-m$ columns and at most $m$ rows; we thus define $f(\pi)=G^{-}$.

Let us now show that the mapping $f$ defined above can be inverted. Let $F$ be a filling of a Ferrers shape with $n-m$ columns and at most $m$ rows. We insert $m-1$ zero columns $c_{2}, c_{3}, \ldots, c_{m}$ into the filling $F$ as follows: each column $c_{i}$ has height $i-1$, and it is inserted immediately after the rightmost column of $F \cup\left\{c_{2}, \ldots, c_{i-1}\right\}$ that has height at most $i-1$. Note that the filling obtained by this operation corresponds to the green cells of the original matrix $M$. Let us call this sparse filling $G$.

We now add a new 1-cell on top of each zero column of $G$, and we add a new 1-cell in front of the bottom row, to obtain a semi-standard filling of a diagram with $n$ columns and $m$ rows. The diagram can be completed into a matrix $M=M(\pi, m)$, where $\pi$ is easily seen to be a canonical sequence of a $\tau$-avoiding partition.

Lemma 9 provides a tool to deal with partition patterns of the form $12 \cdots k(\tau+k) S$ where $S$ is a sequence over $[k]$ and $\tau$ is a partition. We now describe a correspondence between partitions and fillings of stack polyominoes, which is useful for dealing with patterns of the form $12 \cdots k S(\tau+k)$. We use a similar argument as in the proof of Lemma 9.

Lemma 11. If $\tau$ is a partition, and $S$ and $S^{\prime}$ are two nonempty sequences over the alphabet $[k]$ such that $M(S, k) \stackrel{s}{\sim} M\left(S^{\prime}, k\right)$, then the partition $\sigma=12 \cdots k S(\tau+k)$ is equivalent to the partition $\sigma^{\prime}=12 \cdots k S^{\prime}(\tau+k)$.

Proof. Fix a partition $\tau$ with $t$ blocks. Let $\pi$ be any partition of $[n]$ with $m$ blocks, let $M=M(\pi, m)$. We will color the cells of $M$ red and green. A cell of $M$ in row $i$ and column $j$ is green, if it satisfies the following conditions.

(a) The submatrix of $M$ formed by the intersection of the top $m-i$ rows and the rightmost $n-j$ columns contains $M(\tau, t)$.

(b) The matrix $M$ has at least one 1-cell in row $i$ appearing strictly to the left of column $j$.

A cell is called red, if it is not green. Note that the green cells form a stack polyomino and the matrix $M$ induces a sparse filling $G$ of this polyomino. 
As in Lemma 9, it is easy to verify that the partition $\pi$ above avoids the pattern $\sigma$ if and only if the filling $G$ avoids $M(S, k)$, and $\pi$ avoids $\sigma^{\prime}$ if and only if $G$ avoids $M\left(S^{\prime}, k\right)$.

The rest of the argument is analogous to the proof of Lemma 9. Assume that $M(S, k)$ and $M\left(S^{\prime}, k\right)$ are stack-equivalent via a bijection $f$. By Remark 7 , we extend $f$ to a bijection between $M(S, k)$-avoiding and $M\left(S^{\prime}, k\right)$-avoiding sparse fillings of a given stack polyomino. Consider a partition $\pi \in P(n ; \sigma)$ with $m$ blocks, and define $M$ and $G$ as above. Apply $f$ to the filling $G$ to obtain an $M\left(S^{\prime}, k\right)$-avoiding filling $G^{\prime}$; the filling of the red cells of $M$ remains the same. This yields a matrix $M^{\prime}$ and a sequence $\pi^{\prime}$ such that $M^{\prime}=M\left(\pi^{\prime}, k\right)$. We may easily check that the green cells of $M^{\prime}$ are the same as the green cells of $M$. By rule (b) above, the leftmost 1-cell of each row of $M$ is unaffected by this transform. It follows that the first occurrence of $i$ in $\pi^{\prime}$ is at the same place as the first occurrence of $i$ in $\pi$, and in particular, $\pi^{\prime}$ is a partition. By the observation of the previous paragraph, $\pi^{\prime}$ avoids $\sigma^{\prime}$ and the transform $\pi \mapsto \pi^{\prime}$ is a bijection from $P(n ; \sigma)$ to $P\left(n ; \sigma^{\prime}\right)$.

The following simple result about pattern-avoidance in fillings will turn out to be useful in the analysis of pattern avoidance in partitions.

Proposition 12. If $S$ is a nonempty sequence over the alphabet $[k-1]$, then $M(S, k)$ is stack-equivalent to $M(S+1, k)$. If $S$ and $S^{\prime}$ are two sequences over $[k-1]$ such that $M(S, k-1) \stackrel{F}{\sim} M\left(S^{\prime}, k-1\right)$ then $M(S, k) \stackrel{F}{\sim} M\left(S^{\prime}, k\right)$, and if $M(S, k-1) \stackrel{s}{\sim} M\left(S^{\prime}, k-1\right)$ then $M(S, k) \stackrel{s}{\sim} M\left(S^{\prime}, k\right)$.

Proof. To prove the first part, let us define $M=M(S, k), M^{-}=M(S, k-1)$, and $M^{\prime}=M(S+1, k)$. Notice that a filling $F$ of a stack polyomino $\Pi$ avoids $M$ if and only if the filling obtained by erasing the topmost cell of every column of $F$ avoids $M^{-}$. Similarly, $F$ avoids $M^{\prime}$, if and only if the filling obtained by erasing the bottom row of $F$ avoids $M^{-}$. We will now describe a bijection between $M$-avoiding and $M^{\prime}$-avoiding fillings. Fix an $M$-avoiding filling $F$. In every column of this filling, move the topmost element into the bottom row, and move every other element into the row directly above it. This yields an $M^{\prime}$-avoiding filling. The second claim of the theorem is proved analogously.

Note that a sequence $S$ over the alphabet $[k-1]$ does not necessarily contain all the symbols $\{1, \ldots, k-1\}$. In particular, every sequence over $[k-2]$ is also a sequence over $[k-1]$. Thus, if $S$ is a sequence over $[k-2]$, we may use Proposition 12 to deduce $M(S, k) \stackrel{\mathrm{s}}{\sim} M(S+1, k) \stackrel{\mathrm{s}}{\sim} M(S+2, k)$.

For convenience, we translate the first part of Proposition 12 into the language of pattern-avoiding partitions, using Lemma 9 and Lemma 11. We omit the straightforward proof.

Corollary 13. If $S$ is a nonempty sequence over $[k-1]$ and $\tau$ is an arbitrary partition, then

$12 \cdots k(\tau+k) S \sim 12 \cdots k(\tau+k)(S+1)$ and $12 \cdots k S(\tau+k) \sim 12 \cdots k(S+1)(\tau+k)$. 
We now state another result related to pattern-avoidance in Ferrers diagrams, which has important consequences in our study of partitions. Let us first fix the following notation: for two matrices $A$ and $B$, let $\left(\begin{array}{cc}A & 0 \\ 0 & B\end{array}\right)$ denote the matrix with $r(A)+r(B)$ rows and $c(A)+c(B)$ columns with a copy of $A$ in the top left corner and a copy of $B$ in the bottom right corner.

The idea of the following proposition is not new, it has already been applied by Backelin et al. [2] to standard fillings of Ferrers diagrams, and later adapted by de Mier [7] for fillings with arbitrary integers. We now apply it to semi-standard fillings.

Lemma 14. If $A$ and $A^{\prime}$ are two Ferrers equivalent matrices, and if $B$ is an arbitrary matrix, then $\left(\begin{array}{cc}B & 0 \\ 0 & A\end{array}\right) \stackrel{F}{\sim}\left(\begin{array}{cc}B & 0 \\ 0 & A^{\prime}\end{array}\right)$.

Proof. Let $F$ be an arbitrary $\left(\begin{array}{cc}B & 0 \\ 0 & A\end{array}\right)$-avoiding filling of a Ferrers diagram $\Delta$. We say that a cell in row $i$ and column $j$ of $F$ is green if the subfilling of $F$ induced by the intersection of rows $i+1, i+2, \ldots, r(F)$ and columns $1,2, \ldots, j-1$ contains a copy of $B$. Note that the green cells form a Ferrers shape $\Delta^{-} \subseteq \Delta$, and that the restriction of $F$ to the cells of $\Delta^{-}$is a sparse $A$-avoiding filling $G$. By Remark 7 , the filling $G$ can be bijectively transformed into a sparse $A^{\prime}$-avoiding filling $G^{\prime}$ of $\Delta^{-}$, which transforms $F$ into a semi-standard $\left(\begin{array}{cc}B & 0 \\ 0 & A^{\prime}\end{array}\right)$-avoiding filling of $\Delta$.

We remark that the argument of the proof fails if the matrices $\left(\begin{array}{cc}B & 0 \\ 0 & A\end{array}\right)$ and $\left(\begin{array}{cc}B & 0 \\ 0 & A^{\prime}\end{array}\right)$ are replaced with $\left(\begin{array}{cc}A & 0 \\ 0 & B\end{array}\right)$ and $\left(\begin{array}{cc}A^{\prime} & 0 \\ 0 & B\end{array}\right)$ respectively. Also, the argument fails if Ferrers shapes are replaced with stack polyominoes. For instance, the matrix $A=\left(\begin{array}{ll}1 & 0 \\ 0 & 1\end{array}\right)$ is Ferrersequivalent and stack-equivalent to $A^{\prime}=\left(\begin{array}{ll}0 & 1 \\ 1 & 0\end{array}\right)$, but the two matrices $\left(\begin{array}{ll}A & 0 \\ 0 & 1\end{array}\right)$ and $\left(\begin{array}{cc}A^{\prime} & 0 \\ 0 & 1\end{array}\right)$ are not Ferrers-equivalent, and the two matrices $\left(\begin{array}{ll}1 & 0 \\ 0 & A\end{array}\right)$ and $\left(\begin{array}{ll}1 & 0 \\ 0 & A^{\prime}\end{array}\right)$ are not stack-equivalent.

Although Lemma 14 does not directly provide new pairs of equivalent partition patterns, it allows us to prove the following proposition.

Proposition 15. Let $s_{1}>s_{2}>\cdots>s_{m}$ and $t_{1}>t_{2}>\cdots>t_{m}$ be two strictly decreasing sequences over the alphabet $[k]$, let $r_{1}, \ldots, r_{m}$ be positive integers. Define weakly decreasing sequences $S=s_{1}^{r_{1}} s_{2}^{r_{2}} \cdots s_{m}^{r_{m}}$ and $T=t_{1}^{r_{1}} t_{2}^{r_{2}} \cdots t_{m}^{r_{m}}$. We have $M(S, k) \stackrel{F}{\sim} M(T, k)$, and in particular, if $\tau$ an arbitrary partition, then $12 \cdots k(\tau+k) S \sim 12 \cdots k(\tau+k) T$.

Proof. We proceed by induction over minimum $j$ such that $s_{i}=t_{i}$ for each $i \leq m-j$. For $j=0$, we have $S=T$ and the result is clear. If $j>0$, assume without loss of generality that $s_{m-j+1}-t_{m-j+1}=d>0$. Consider the sequence $t_{1}^{\prime}>t_{2}^{\prime}>\cdots>t_{m}^{\prime}$ such that $t_{i}^{\prime}=t_{i}$ for every $i \leq m-j$ and $t_{i}^{\prime}=t_{i}+d$ for every $i>m-j$. The sequence $\left(t_{i}^{\prime}\right)_{i=1}^{m}$ is strictly decreasing, and its first $m-j+1$ terms are equal to $s_{i}$. Define $T^{\prime}=\left(t_{1}^{\prime}\right)^{r_{1}}\left(t_{2}^{\prime}\right)^{r_{2}} \cdots\left(t_{m}^{\prime}\right)^{r_{m}}$. By induction, $M(S, k) \stackrel{\mathrm{F}}{\sim} M\left(T^{\prime}, k\right)$. To prove that $M(T, k) \stackrel{\mathrm{F}}{\sim} M\left(T^{\prime}, k\right)$, first write $T=$ $T_{0} T_{1}$, where $T_{0}$ is the prefix of $T$ containing all the symbols of $T$ greater than $t_{m-j+1}$ and $T_{1}$ is the suffix of the remaining symbols. Notice that $T^{\prime}=T_{0}\left(T_{1}+d\right)$. We may write $M(T, k)=\left(\begin{array}{cc}B & 0 \\ 0 & A\end{array}\right)$ and $M\left(T^{\prime}, k\right)=\left(\begin{array}{cc}B & 0 \\ 0 & A^{\prime}\end{array}\right)$, where $A=M\left(T_{1}, t_{m-j}-1\right)$ and $A^{\prime}=$ $M\left(T_{1}+d, t_{m-j}-1\right)$. By Proposition $12, A \stackrel{\mathrm{F}}{\sim} A^{\prime}$, and by Lemma $14, M(T, k) \stackrel{\mathrm{F}}{\sim} M\left(T^{\prime}, k\right)$, as claimed. The last claim of the proposition follows from Lemma 9. 


\subsection{Non-crossing and non-nesting partitions}

The key application of the framework of the previous subsection is the identity between non-crossing and non-nesting partitions. We define non-crossing and non-nesting partitions in the following way.

Definition 16. A partition is $k$-noncrossing if it avoids the pattern $12 \cdots k 12 \cdots k$, and it is $k$-nonnesting if it avoids the pattern $12 \cdots k k(k-1) \cdots 1$.

Let us point out that there are several different concepts of 'crossings' and 'nestings' used in the literature: for example, Klazar [13] has considered two blocks $X, Y$ of a partition to be crossing (or nesting) if there are four elements $x_{1}<y_{1}<x_{2}<y_{2}$ (or $x_{1}<y_{1}<y_{2}<x_{2}$, respectively) such that $x_{1}, x_{2} \in X$ and $y_{1}, y_{2} \in Y$, and similarly for $k$-crossings and $k$-nestings. Unlike our approach, Klazar's definition makes no assumption about the relative order of the minimal elements of $X$ and $Y$, which allows more general configurations to be considered as crossing or nesting. Thus, Klazar's $k$-noncrossing and $k$-nonnesting partitions are a proper subset of our $k$-noncrossing and $k$-nonnesting partitions, (except for 2-noncrossing partitions where the two concepts coincide).

Another approach to crossings in partitions has been pursued by Chen et al. [3, 4]. They use the so-called linear representation, where a partition of $[n]$ with blocks $B_{1}, B_{2}, \ldots, B_{k}$ is represented by a graph on the vertex set $[n]$, with $a, b \in[n]$ connected by an edge if they belong to the same block and there is no other element of this block between them. In this terminology, a partition is $k$-crossing (or $k$-nesting) if the representing graph contains $k$ edges which are pairwise crossing (or nesting), where two edges $e_{1}=\{a<b\}$ and $e_{2}=\left\{a^{\prime}<b^{\prime}\right\}$ are crossing (or nesting) if $a<a^{\prime}<b<b^{\prime}$ (or $a<a^{\prime}<b^{\prime}<b$ respectively). Let us call such partitions graph- $k$-crossing and graph- $k$-nesting, to avoid confusion with our own terminology of Definition 16. It is not difficult to see that a partition is graph-2-noncrossing if and only if it is 2-noncrossing, but for nestings and for $k$ crossings with $k>2$, the two concepts are incomparable. For instance the partition 12121 is graph-2-nonnesting but it contains 1221, while 12112 is graph-2-nesting and avoids 1221. Similarly, 1213123 has no graph-3-crossing and contains 123123 , while 1232132 has a graph-3-crossing and avoids 123123 .

Chen et al. [4] have shown that the number of graph- $k$-noncrossing and graph- $k$ nonnesting partitions of $[n]$ is equal. Below, we prove that the same is true for $k$ noncrossing and $k$-nonnesting partitions as well. It is interesting to note that the proofs of both these results are based on a reduction to theorems on pattern avoidance in the fillings of Ferrers diagrams (this is only implicit in [4], a direct construction is given by Krattenthaler [15]), although the constructions employed in the proofs of these results are quite different.

Theorem 17. For every $n$ and $k$, the number of $k$-noncrossing partitions of $[n]$ is equal to the number of $k$-nonnesting partitions of $[n]$.

By Lemma 9, a bijection between $k$-noncrossing and $k$-nonnesting partitions can be constructed from a bijection between $I_{k}$-avoiding and $J_{k}$-avoiding semi-standard fillings of Ferrers diagrams. 
Krattenthaler [15] has presented a comprehensive summary of the relationships between $I_{r}$-avoiding and $J_{r}$-avoiding fillings of a fixed Ferrers diagram under additional constraints for row-sums and column-sums. These relationships are based on a suitable version of the RSK-correspondence (see [10] or [25] for a broad overview of the RSK algorithm and related topics).

We will now state the theorem about the correspondence between $I_{k}$-avoiding and $J_{k}$-avoiding fillings of diagrams. The result we will use is a weaker version of Theorem 13 from [15]. Note that in the original paper, it is not explicitly stated that the bijection between $I_{k}$-avoiding and $J_{k}$-avoiding fillings preserves the sum of every row and every column; however, this is an immediate consequence of the technique used in the proof. Also, in [15], the result is stated for arbitrary fillings with nonnegative integers; however, the previous remark shows that the result holds even when restricted to semi-standard fillings.

Theorem 18 (adapted from [15]). For every Ferrers diagram $\Delta$ and every $k$, there is a bijection between the $I_{k}$-avoiding semi-standard fillings of $\Delta$ and the $J_{k}$-avoiding semistandard fillings of $\Delta$. The bijection preserves the number of 1-cells in every row.

Theorem 18 and Lemma 9 give us the result we need. We even obtain the following refinement of Theorem 17 .

Corollary 19. For every $n$ and every $k$, there is a bijection between $k$-noncrossing and $k$-nonnesting partitions of $[n]$. The bijection preserves the number of blocks, the size of each block, and the smallest element of every block.

Applying Lemma 9 with $S=12 \cdots k$ and $S^{\prime}=k(k-1) \cdots 1$, and translating it into the terminology of pattern-avoiding partitions, we obtain the following result.

Corollary 20. Let $\tau$ be a partition, let $k$ be an integer. The pattern $12 \cdots k(\tau+k) 12 \cdots k$ is equivalent to $12 \cdots k(\tau+k) k(k-1) \cdots 1$.

Furthermore, results of Rubey, in particular [20, Proposition 5.3], imply that the matrices $I_{k}$ and $J_{k}$ are in fact stack-equivalent, rather than just Ferrers-equivalent. More precisely, Rubey's theorem deals with fillings of moon polyominoes with prescribed rowsums. However, since a transposed copy of a stack polyomino is a special case of a moon polyomino, Rubey's general result applies to fillings of stack polyominoes with prescribed column sums as well. Combining this theorem with Lemma 11, we obtain the following result.

Corollary 21. For any $k$ and any partition $\tau$, the pattern $12 \cdots k 12 \cdots k(\tau+k)$ is equivalent to $12 \cdots k k(k-1) \cdots 1(\tau+k)$.

\subsection{The patterns $12 \cdots k(k+1) 12 \cdots k$ and $12 \cdots k 12 \cdots k(k+1)$}

Our next aim is to prove that the pattern $12 \cdots k(k+1) 12 \cdots k$ is equivalent to the pattern $12 \cdots k 12 \cdots k(k+1)$. This result is again a consequence of earlier results on fillings of polyominoes. 
Definition 22. Let $\Pi$ be a stack polyomino. The content of $\Pi$ is the sequence of the column heights of $\Pi$, listed in nondecreasing order.

The key ingredient of our proof is the following result of Rubey.

Theorem 23. Let $\Pi$ and $\Pi$ ' be two stack polyominoes with the same content, and let $k \geq 1$ be an integer. There is a bijection between the $I_{k}$-avoiding semi-standard fillings of $\Pi$ and the $I_{k}$-avoiding semi-standard fillings of $\Pi^{\prime}$.

The theorem above is essentially a special case of Proposition 5.3 from Rubey's paper [20]. The only complication is that Rubey's proposition deals with arbitrary nonnegative integer fillings, rather than semi-standard fillings. However, as was pointed out in the last paragraph of Section 4 in [20], it is easy to see that Rubey's bijection maps semi-standard fillings to semi-standard fillings.

Observe that Theorem 23 implies that $I_{k}$ and $J_{k}$ are stack-equivalent. The number of $J_{k}$-avoiding fillings of a stack polyomino $\Sigma$ is clearly equal to the number of $I_{k}$-avoiding fillings of the mirror image of $\Sigma$, which is equal to the number of $I_{k}$-avoiding fillings of $\Sigma$ by Theorem 23 .

Let us now analyze in more detail the partitions avoiding $12 \cdots k(k+1) 12 \cdots k$.

Definition 24. Let $\pi=\pi_{1} \cdots \pi_{n}$ be a partition. We say that an element $\pi_{i}$ is leftdominating if $\pi_{i} \geq \pi_{j}$ for each $j<i$. We say that a left-dominating element $\pi_{i}$ leftdominates an element $\pi_{j}$, if $\pi_{i}>\pi_{j}, i<j$, and $\pi_{i}$ is the rightmost left-dominating element with these two properties. Clearly, if $\pi_{j}$ not left-dominating, then it is leftdominated by a unique left-dominating element. On the other hand, a left-dominating element is not left-dominated by any other element. If an element is not left-dominating, we call it simply left-dominated.

The left shadow of $\pi$ is the sequence $\bar{\pi}$ obtained by replacing each left-dominated element by the symbol ' $*$ '. We will say that a non-star symbol $i$ left-dominates an occurrence of a star, if $i$ is the rightmost non-star to the left of the star.

For example, if $\pi=123232144$, the left shadow of $\pi$ is the sequence $\bar{\pi}=123 * 3 * * 44$. In $\bar{\pi}$, the leftmost occurrence of ' 3 ' left-dominates a single star, while the second occurrence of ' 3 ' left-dominates two stars.

It is not difficult to see that a sequence $\bar{\pi}$ over the alphabet $\{1,2, \ldots, m, *\}$ is a left shadow of a partition with $m$ blocks if and only if it satisfies the following conditions.

- The non-star symbols of $\bar{\pi}$ form a non-decreasing sequence.

- Each of the symbols $1,2, \ldots, m$ appears at least once.

- No occurrence of the symbol 1 may left-dominate an occurrence of $*$. Any other non-star symbol may left-dominate any number of stars, and each star is dominated by a non-star.

Any sequence that satisfies these three conditions will be called a left-shadow sequence. Note that a left-shadow sequence is uniquely determined by the multiplicities of its nonstar symbols and by the number of stars dominated by each non-star. 
Definition 25. Let $\pi=\pi_{1} \cdots \pi_{n}$ be a partition, let $F=F(\pi)$ be the semi-standard filling of a Ferrers diagram defined by the following conditions.

1. The columns of $F$ correspond to the left-dominated elements of $\pi$. The $i$-th column of $F$ has height $j$ if the $i$-th left-dominated element of $\pi$ is dominated by an occurrence of $j+1$.

2. The $i$-th column of $F$ has a 1 -cell in row $j$ if the $i$-th left-dominated element of $\pi$ is equal to $j$.

Note that the shape of the underlying diagram of $F(\pi)$ is determined by the left shadow of $\pi$. More precisely, the number of columns of height $h$ in $F$ is equal to the number of stars in the left shadow which are dominated by an occurrence of $h+1$. It is easy to see that the left shadow $\bar{\pi}$ and the filling $F(\pi)$ together uniquely determine the partition $\pi$. In fact, for every semi-standard filling $F^{\prime}$ with the same shape as $F(\pi)$, there is a (unique) partition $\pi^{\prime}$ with the same left-shadow as $\pi$, and with $F\left(\pi^{\prime}\right)=F^{\prime}$.

The following observation is a straightforward application of the terminology introduced above. We omit its proof.

Observation 26. A partition $\pi$ avoids the pattern $12 \cdots k(k+1) 12 \cdots k$ if and only if the filling $F(\pi)$ avoids $I_{k}$.

We now focus on the partitions that avoid the pattern $12 \cdots k 12 \cdots k(k+1)$.

Definition 27. Let $\pi=\pi_{1} \cdots \pi_{n}$ be a partition. We say that an element $\pi_{i}$ is rightdominating if either $\pi_{i} \geq \pi_{j}$ for each $j>i$ or $\pi_{i}>\pi_{j}$ for each $j<i$. If $\pi_{i}$ is not right-dominating, we say that it is right-dominated. We say that $\pi_{i}$ right-dominates $\pi_{j}$ if $\pi_{i}$ is the leftmost right-dominating element appearing to the right of $\pi_{j}$, and $\pi_{j}$ itself is not right-dominating.

The right shadow $\widetilde{\pi}$ of a partition $\pi$ is obtained by replacing each right-dominated element of $\pi$ by a star.

For example, the right shadow of the partition $\pi=12213423312$ is the sequence $12 * * 34 * 33 * 2$. A sequence $\widetilde{\pi}$ over the alphabet $\{1,2, \ldots, m, *\}$ is the right shadow of a partition with $m$ blocks if and only if it satisfies the following conditions.

- The non-star symbols of $\widetilde{\pi}$ form a subsequence $\left(1,2, \ldots, m, s_{1}, s_{2}, \ldots, s_{p}\right)$ where the sequence $s_{1} s_{2} \cdots s_{p}$ is nonincreasing.

- No occurrence of the symbol 1 may right-dominate an occurrence of $*$. Any other non-star symbol may right-dominate any number of stars, and each star is rightdominated by a non-star.

Any sequence that satisfies these two conditions will be called a right-shadow sequence. A right-shadow sequence is uniquely determined by the multiplicities of its non-star symbols and by the number of stars right-dominated by each non-star. 
Definition 28. Let $\pi=\pi_{1} \cdots \pi_{n}$ be a partition. Let $S=S(\pi)$ be the semi-standard filling of a stack polyomino defined by the following conditions.

1. The columns of $S$ correspond to the right-dominated elements of $\pi$. The $i$-th column of $S$ has height $j$ if the $i$-th right-dominated element of $\pi$ is dominated by an occurrence of $j+1$.

2. The $i$-th column of $S$ has a 1-cell in row $j$ if the $i$-th right-dominated element of $\pi$ is equal to $j$.

Let $\Sigma$ be the underlying diagram of $S(\pi)$. Notice that $\Sigma$ is uniquely determined by the right shadow $\tilde{\pi}$ of the partition $\pi$, although there may be different right shadows corresponding to the same shape $\Sigma$. The sequence $\tilde{\pi}$ and the filling $S(\pi)$ together determine the partition $\pi$. For a fixed $\widetilde{\pi}$, the mapping $\pi \mapsto S(\pi)$ gives a bijection between partitions with right shadow $\tilde{\pi}$ and fillings of $\Sigma$.

The proof of the following observation is again straightforward and we omit it.

Observation 29. A partition $\pi$ avoids the pattern $12 \cdots k 12 \cdots k(k+1)$ if and only if the flling $S(\pi)$ avoids $I_{k}$.

We are now ready to prove the main result of this subsection.

Theorem 30. For any $k \geq 1$, the patterns $12 \cdots k(k+1) 12 \cdots k$ and $12 \cdots k 12 \cdots k(k+1)$ are equivalent.

Proof. We will describe a bijection between the two pattern-avoiding classes. Let $\pi$ be a partition with $m$ blocks that avoids $12 \cdots k(k+1) 12 \cdots k$. Let $\bar{\pi}$ be its left shadow, and let $F(\pi)$ be the filling from Definition 25. Let $\Pi$ denote the underlying shape of $F(\pi)$. By Observation 26, $F(\pi)$ avoids $I_{k}$.

Let $\widetilde{\sigma}$ be the right-shadow sequence determined by the following two conditions.

1. For each symbol $i \in[m]$, the number of occurrences of $i$ in $\bar{\pi}$ is equal to the number of its occurrences in $\widetilde{\sigma}$.

2. For any $i$ and $j$, the number of stars left-dominated by the $j$-th occurrence of $i$ in $\bar{\pi}$ is equal to the number of stars right-dominated by the $j$-th occurrence of $i$ in $\tilde{\sigma}$.

Note that these conditions determine $\widetilde{\sigma}$ uniquely. As an example, consider the left-shadow sequence $\bar{\pi}=123 * 3 * * 44 *$. In $\widetilde{\sigma}$, the non-star elements form the subsequence 123443 . The first occurrence of 3 in $\bar{\pi}$ left-dominates a single star, the second occurrence of 3 left-dominates two stars, and the second occurrence of 4 left-dominates one star. Hence, $\widetilde{\sigma}$ is the sequence $12 * 34 * 4 * * 3$.

Next, let $\Sigma$ be the stack polyomino whose columns correspond to the stars of $\widetilde{\sigma}$, where the $i$-th column has height $h$ if the $i$-th star of $\widetilde{\sigma}$ is right-dominated by $h+1$. In the example above, if $\widetilde{\sigma}=12 * 34 * 4 * * 3$, then $\Sigma$ has four columns of heights $(2,3,2,2)$. Clearly, $\Sigma$ has the same content as $\Pi$. By Theorem 23, there is a bijection $f$ between the $I_{k}$-avoiding fillings of $\Pi$ and the $I_{k}$-avoiding fillings of $\Sigma$. This bijection transforms $F(\pi)$ 
into a filling $S$ of $\Sigma$. Define a partition $\sigma$ by replacing the $i$-th star in $\widetilde{\sigma}$ by the row-index of the 1-cell in the $i$-th column of $S$. By construction, $\sigma$ is a partition with right shadow $\tilde{\sigma}$, and $S(\sigma)=S$. By Observation $29, \sigma$ avoids $12 \cdots k 12 \cdots k(k+1)$.

This transformation, which is easily seen to be invertible, provides the required bijection. This completes the proof.

\subsection{Patterns of the form $1(\tau+1)$}

In this subsection, we will establish a general relationship between the partitions that avoid a pattern $\tau$ and the partitions that avoid the pattern $1(\tau+1)$. The key result is the following theorem.

Theorem 31. Let $\tau$ be an arbitrary pattern, and let $F(x)$ be its corresponding EGF. Let $\sigma=1(\tau+1)$, and let $G(x)$ be its $E G F$. For every $n \geq 1$, the following holds:

$$
p(n ; \sigma)=\sum_{i=0}^{n-1}\left(\begin{array}{c}
n-1 \\
i
\end{array}\right) p(i ; \tau) .
$$

In terms of generating functions, this is equivalent to

$$
G(x)=1+\int_{0}^{x} F(t) e^{t} d t
$$

Proof. Fix $\sigma$ and $\tau$ as in the statement of the theorem. Let $\pi$ be an arbitrary partition, and let $\pi^{-}$denote the partition obtained from $\pi$ by erasing every occurrence of the symbol 1 , and decreasing every other symbol by 1 ; in other words, $\pi^{-}$represents the partition obtained by removing the first block from the partition $\pi$. Clearly, a partition $\pi$ avoids $\sigma$ if and only if $\pi^{-}$avoids $\tau$. Thus, for every $\sigma$-avoiding partition $\pi \in P(n ; \sigma)$ there is a unique $\tau$-avoiding partition $\rho \in \cup_{i=0}^{n-1} P(i ; \tau)$ satisfying $\pi^{-}=\rho$. On the other hand, for a fixed $\rho \in P(i ; \tau)$, there are $\left(\begin{array}{c}n-1 \\ i\end{array}\right)$ partitions $\pi \in P(n ; \sigma)$ such that $\pi^{-}=\rho$. This gives equation (3).

To get equation (4), we multiply both sides of (3) by $\frac{x^{n}}{n !}$ and sum for all $n \geq 1$. This yields

$$
\begin{aligned}
G(x)-1 & =\sum_{n \geq 1} \frac{x^{n}}{n !} \sum_{i=0}^{n-1}\left(\begin{array}{c}
n-1 \\
i
\end{array}\right) p(i ; \tau)=\int_{0}^{x} \sum_{n \geq 1} \frac{t^{n-1}}{(n-1) !} \sum_{i=0}^{n-1}\left(\begin{array}{c}
n-1 \\
i
\end{array}\right) p(i ; \tau) \mathrm{d} t \\
& =\int_{0}^{x} \sum_{n \geq 0} \frac{t^{n}}{n !} \sum_{i=0}^{n}\left(\begin{array}{c}
n \\
i
\end{array}\right) p(i ; \tau) \mathrm{d} t=\int_{0}^{x} \sum_{n \geq 0} \sum_{i=0}^{n} \frac{t^{i}}{i !} p(i ; \tau) \frac{t^{n-i}}{(n-i) !} \mathrm{d} t \\
& =\int_{0}^{x}\left(\sum_{i \geq 0} \frac{t^{i}}{i !} p(i ; \tau)\right)\left(\sum_{k \geq 0} \frac{t^{k}}{k !}\right) \mathrm{d} t=\int_{0}^{x} F(t) e^{t} \mathrm{~d} t,
\end{aligned}
$$

which is equivalent to equation (4). 
The following result is an immediate consequence of Theorem 31 .

Corollary 32. If $\tau \sim \tau^{\prime}$ then $1(\tau+1) \sim 1\left(\tau^{\prime}+1\right)$, and more generally, $12 \cdots k(\tau+k) \sim$ $12 \cdots k\left(\tau^{\prime}+k\right)$. In particular, since $123 \sim 122 \sim 112 \sim 121$, we see that for every $m \geq 2$ the patterns $12 \cdots(m-1) m(m+1), 12 \cdots(m-1) m m, 12 \cdots(m-1)(m-1) m$ and $12 \cdots(m-1) m(m-1)$ are equivalent. Conversely, if $1(\tau+1) \sim 1\left(\tau^{\prime}+1\right)$, then $\tau \sim \tau^{\prime}$.

Proof. To prove the last claim, notice that equation (3) can be inverted to obtain

$$
p(n-1 ; \tau)=\sum_{i=0}^{n-1}(-1)^{i}\left(\begin{array}{c}
n-1 \\
i
\end{array}\right) p(n-i ; \sigma) .
$$

The other claims follow directly from Theorem 31.

\subsection{Patterns equivalent to $12 \cdots m(m+1)$}

The partitions that avoid $12 \cdots m(m+1)$, or equivalently, the partitions with at most $m$ blocks, are a very natural pattern-avoiding class of partitions. Their number may be expressed by $p(n ; 12 \cdots(m+1))=\sum_{i=0}^{m} S(n, i)$, where $S(n, i)$ is the Stirling number of the second kind, which is equal to the number of partitions of $[n]$ with exactly $i$ blocks.

As an application of the previous results, we will now present two classes of patterns that are equivalent to the pattern $12 \cdots(m+1)$. From this result, we obtain an alternative combinatorial interpretation of the Stirling numbers $S(n, i)$.

Our result is summarized in the following theorem.

Theorem 33. For every $m \geq 2$, the following patterns are equivalent:

(a) $12 \cdots(m-1) m(m+1)$,

(b) $12 \cdots(m-1) m d$, where $d$ is any number from the set $[m]$,

(c) $12 \cdots(m-1) d m$, where $d$ is any number from the set $[m-1]$.

Proof. From Corollary 32, we get the equivalences

$$
12 \cdots m(m+1) \sim 12 \cdots(m-1) m m \sim 12 \cdots(m-1)(m-1) m .
$$

The equivalences

$$
12 \cdots(m-1) m m \sim 12 \cdots(m-1) m d \text { and } 12 \cdots(m-1)(m-1) m \sim 12 \cdots(m-1) d m
$$

are obtained by a repeated application of Corollary 13. 


\subsection{Binary patterns}

Let us now focus on the avoidance of binary patterns, i.e., the patterns that only contain the symbols 1 and 2 .

We will first consider the forbidden patterns of the form $1^{k} 21^{\ell}$. We have already seen that $112 \sim 121$. The following theorem offers a generalization.

Theorem 34. For any three integers $j, k, m$ satisfying $1 \leq j, k \leq m$, the pattern $1^{j} 21^{m-j}$ is equivalent to the pattern $1^{k} 21^{m-k}$.

Before we present the proof of Theorem 34, we need some preparation. Let $\pi=$ $\pi_{1} \pi_{2} \cdots \pi_{n}$ be a partition. Clearly, $\pi$ can be uniquely expressed as $1 P_{1} 1 P_{2} 1 \cdots 1 P_{t-1} 1 P_{t}$, where the $P_{i}$ are (possibly empty) maximal contiguous subsequences of $\pi$ that do not contain the symbol 1 . The sequence $P_{i}$ will be referred to as the $i$-th chunk of $\pi$. By concatenating the chunks into a sequence $P=P_{1} \cdots P_{t}$ and then subtracting 1 from every symbol of $P$, we obtain a canonical sequence of a partition; let this partition be denoted by $\pi^{-}$. The key ingredient in the proof of Theorem 34 is the following lemma.

Lemma 35. Let $\pi$ be a partition that has $t$ occurrences of the symbol 1, let $P_{i}$ and $\pi^{-}$be as above. Let $j \geq 1$ and $k \geq 0$ be two integers. The partition $\pi$ avoids $1^{j} 21^{k}$ if and only if the following two conditions hold.

- The partition $\pi^{-}$avoids $1^{j} 21^{k}$.

- For every $i$ such that $j \leq i \leq t-k$, the chunk $P_{i}$ is empty.

Proof. Clearly, the two conditions are necessary. To see that they are sufficient, we argue by contradiction. Let $\pi$ be a partition that satisfies the two conditions, and assume that $\pi$ has a subsequence $a^{j} b a^{k}$ for two symbols $a<b$. If $a=1$ we have a contradiction with the second condition, and if $a>1$, then $\pi^{-}$contains the sequence $(a-1)^{j}(b-1)(a-1)^{k}$, contradicting the first condition.

We are now ready prove Theorem 34 .

Proof of Theorem 34. It is enough to prove that for every $k \geq 1$ and every $m>k$ there is a bijection $f$ from $P\left(n ; 1^{k} 21^{m-k}\right)$ to $P\left(n ; 1^{m} 2\right)$. To define $f$, we will proceed by induction on the number of blocks of $\pi$. If $\pi=1^{n}$ then we define $f(\pi)=\pi$. Assume that $f$ has been defined for all partitions with fewer than $b$ blocks, and let $\pi \in P\left(n ; 1^{k} 21^{m-k}\right)$ be a partition with $b$ blocks, let $t$ be the size of the first block of $\pi$. Let $P_{1}, \ldots, P_{t}$ be the chunks of $\pi$ and let $\pi^{-}$be defined as above. Define $\bar{\sigma}=f\left(\pi^{-}\right)$. This is well defined, since $\pi^{-} \in P\left(n-t ; 1^{k} 21^{m-k}\right)$ and $\pi^{-}$has $b-1$ blocks. Let $S=\bar{\sigma}+1$. We express $S$ as a concatenation of the form $S=S_{1} S_{2} \cdots S_{t}$, where the length of $S_{i}$ is equal to the length of $P_{i}$. By Lemma 35, the chunk $P_{i}$ (and hence also $S_{i}$ ) is empty whenever $k \leq i \leq t-m+k$. We put $f(\pi)=\sigma$, where $\sigma$ is defined as follows.

- If $t<m$, then $\sigma=1 S_{1} 1 S_{2} 1 \cdots 1 S_{t-1} 1 S_{t}$. 
- If $t \geq m$, then $\sigma=1 S_{1} 1 S_{2} 1 \cdots 1 S_{k-1} 1 S_{t-m+k+1} 1 S_{t-m+k+2} 1 \cdots 1 S_{t-1} 1 S_{t} 1^{t-m+1}$.

Using Lemma 35, we may easily see that $\sigma$ avoids $1^{m} 2$. It is also straightforward to check that $f$ is indeed a bijection from $P\left(n ; 1^{k} 21^{m-k}\right)$ to $P\left(n ; 1^{m} 2\right)$. Note that $f$ preserves not only the number of blocks of the partition, but also the size of each block.

Using our results on fillings, we can add another pattern to the equivalence class covered by Theorem 34 .

Theorem 36. For every $m \geq 1$, the pattern $12^{m}$ is equivalent to the pattern $121^{m-1}$.

Proof. This is just Corollary 13 with $k=2$ and $S=1^{m-1}$.

Corollary 37. Let $m$ be a positive integer, let $\tau$ be any pattern from the set

$$
T=\left\{1^{k} 21^{m-k}: 1 \leq k \leq m\right\} \cup\left\{12^{m}\right\} .
$$

The EGF $F(x)$ of a pattern $\tau \in T$ is given by

$$
F(x)=1+\int_{0}^{x} \exp \left(t+\sum_{i=1}^{m-1} \frac{t^{i}}{i !}\right) d t
$$

Proof. Theorems 34 and 36 show that all the patterns from the set $\mathrm{T}$ are equivalent, so we will compute the EGF of $\tau=12^{m}$. The formula for $F(x)$ follows directly from equation (1) on page 4 and Theorem 31.

We now turn to another type of binary patterns, namely the patterns of the form $12^{k} 12^{m-k}$ with $1 \leq k \leq m$. For a fixed $m$, these patterns are all equivalent. To prove this, it suffices to show that the matrices $M\left(2^{k-1} 12^{m-k}, 2\right)$ are all Ferrers-equivalent, and then apply Lemma 9. We will construct a bijection between pattern-avoiding fillings which proves the Ferrers-equivalence of these matrices. Furthermore, we will show that this bijection has additional properties, which will be useful in proving more complicated criteria for partition-equivalence that cannot be obtained from Lemma 9 alone.

Definition 38. Let $F$ be a sparse filling of a stack polyomino $\Pi$ and let $t \geq 1$ be an integer. A sequence $c_{1}, c_{2}, \ldots, c_{t}$ of 1-cells in $F$ is called a decreasing chain if for every $i \in[t-1]$ the column containing $c_{i}$ is to left of the column containing $c_{i+1}$ and the row containing $c_{i}$ is above the row of $c_{i+1}$. An increasing chain is defined analogously.

A filling is $t$-falling if it has at least $t$ rows, and in its bottom $t$ rows, the leftmost 1-cells of the nonzero rows form a decreasing chain.

Notice that a $t$-falling semi-standard filling of a stack polyomino $\Pi$ only exists if the leftmost column of $\Pi$ has height at least $t$.

In the rest of this subsection, $S_{q}^{p}$ denotes the sequence $2^{p} 12^{q}$ and $\bar{S}_{q}^{p}$ denotes the sequence $1^{p} 21^{q}$, where $p, q$ are nonnegative integers. 
Lemma 39. For every $p, q \geq 0$, the matrix $M\left(S_{q}^{p}, 2\right)$ is stack-equivalent to the matrix $M\left(S_{0}^{p+q}, 2\right)$. Furthermore, if $p \geq 1$, then for every stack polyomino $\Pi$, there is a bijection $f$ between the $M\left(S_{q}^{p}, 2\right)$-avoiding and $M\left(S_{0}^{p+q}, 2\right)$-avoiding semi-standard fillings of $\Pi$ with the following properties.

- The bijection $f$ preserves the number of 1-cells in every row.

- Both $f$ and $f^{-1}$ map $t$-falling fillings to $t$-falling fillings, for every $t \geq 1$.

Proof. Let $M=M\left(S_{q}^{p}, 2\right)$ and $M^{\prime}=M\left(S_{0}^{p+q}, 2\right)$, for some $p, q \geq 0$. Let $\Pi$ be a stack polyomino. We will proceed by induction over the number of rows of $\Pi$. If $\Pi$ has only one row, then a constant mapping is the required bijection. Assume now that $\Pi$ has $r \geq 2$ rows, and assume that we are presented with a semi-standard filling $F$ of $\Pi$. Let $\Pi^{-}$be the diagram obtained from $\Pi$ by erasing the $r$-th row as well as every column that contains a 1-cell of $F$ in the $r$-th row. The filling $F$ induces on $\Pi^{-}$a semi-standard filling $F^{-}$.

We claim that for every $p, q \geq 0$, a filling $F$ avoids $M$ if and only if the following two conditions are satisfied.

(a) The filling $F^{-}$avoids $M$.

(b) If the $r$-th row of $F$ contains $m$ 1-cells in columns $c_{1}<c_{2}<\cdots<c_{m}$ and if $m \geq p+q$, then for every $i$ such that $p \leq i \leq m-q$, the column $c_{i}$ is either the rightmost column of the $r$-th row of $\Pi$, or it is directly adjacent to the column $c_{i+1}$ (i.e. $c_{i}+1=c_{i+1}$ ).

Clearly, the two conditions are necessary. We now show that they are sufficient. The first condition guarantees that $F$ does not contain any copy of $M$ that would be confined to the first $r-1$ rows. The second condition guarantees that $F$ has no copy of $M$ that would intersect the $r$-th row.

We now define recursively the required bijection between $M$-avoiding and $M^{\prime}$-avoiding fillings. Let $F$ be an $M$-avoiding filling of $\Pi$, let $F^{-}$and $c_{1}, \ldots, c_{m}$ be as above. By the induction hypothesis, we already have a bijection between $M$-avoiding and $M^{\prime}$-avoiding fillings of the shape $\Pi^{-}$. This bijection maps $F^{-}$to a filling $\tilde{F}^{-}$of $\Pi^{-}$. Let $\tilde{F}$ be the filling of $\Pi$ that has the same values as $F$ in the $r$-th row, and the columns not containing a 1-cell in the $r$-th row are filled according to $\tilde{F}^{-}$. Note that $\tilde{F}$ contains no copy of $M^{\prime}$ in its first $r-1$ rows and it contains no copy of $M$ that would intersect the $r$-th row.

If $\tilde{F}$ has fewer than $p+q$ 1-cells in the $r$-th row, we define $f(F)=\tilde{F}$, otherwise we modify $\tilde{F}$ in the following way. For every $i=1, \ldots, q$, we consider the columns with indices strictly between $c_{m-q+i}$ and $c_{m-q+i+1}$ (if $i=q$, we take all columns to the right of $c_{m}$ that intersect the last row). We remove these columns from $\tilde{F}$ and re-insert them between the columns $c_{p+i-1}$ and $c_{p+i}$ (which used to be adjacent by condition (b) above). Note that these transformations preserve the relative left-to-right order of all the columns that do not contain a 1-cell in their $r$-th row. In particular, the resulting filling still has no copy of $M^{\prime}$ in the first $r-1$ rows. By construction, the filling also satisfies condition (b) for the values $p^{\prime}=p+q$ and $q^{\prime}=0$ used instead of the original $p$ and $q$. Hence, it is 
a $M^{\prime}$-avoiding filling. This construction provides a bijection $f$ between $M$-avoiding and $M^{\prime}$-avoiding fillings.

It is clear that $f$ preserves the number of 1-cells in each row. It remains to check that if $p \geq 1$, then $f$ preserves the $t$-falling property. Let us fix $t$, and let $r$ be the number of rows of $\Pi$. If $r<t$ then no filling of $\Pi$ is $t$-falling. If $r=t$, then $F$ is $t$-falling if and only if $F^{-}$is $(t-1)$-falling and the $r$-th row is either empty or has a 1-cell in the leftmost column of $\Pi$. These conditions are preserved by $f$ and $f^{-1}$, provided $p \geq 1$. Finally, if $r>t$, then $F$ is $t$-falling if and only if $F^{-}$is $t$-falling. We now obtain the required result from the induction hypothesis and from the fact that the relative position of the 1-cells of the first $r-1$ rows does not change when we transform $\tilde{F}$ into $f(F)$.

With the help of Lemma 39, we are able to prove several results about pattern avoidance in partitions. We first prove a direct corollary of previous results.

Corollary 40. For any partition $\tau$, for any $k \geq 2$, and for any $p, q \geq 0$, the pattern $12 \cdots k(\tau+k) S_{q}^{p}$ is equivalent to $12 \cdots k(\tau+k) S_{0}^{p+q}$, and $12 \cdots k S_{q}^{p}(\tau+k)$ is equivalent to $12 \cdots k S_{0}^{p+q}(\tau+k)$.

Proof. By Lemma 39, the two matrices $M\left(S_{q}^{p}, 2\right)$ and $M\left(S_{0}^{p+q}, 2\right)$ are Ferrers-equivalent. By Proposition 12, this implies that $M\left(S_{q}^{p}, k\right) \stackrel{\mathrm{F}}{\sim} M\left(S_{0}^{p+q}, k\right)$ for any $k \geq 2$. Lemma 9 then gives the first equivalence. The second equivalence follows from Lemma 11 by an analogous argument.

Next, we present two theorems that make use of the $t$-falling property. Recall that $\bar{S}_{q}^{p}=1^{p} 21^{q}$.

Theorem 41. Let $\tau$ be any partition with $k$ blocks, let $p \geq 1$ and $q \geq 0$. The pattern $\sigma=\tau\left(\bar{S}_{q}^{p}+k\right)$ is equivalent to $\sigma^{\prime}=\tau\left(\bar{S}_{0}^{p+q}+k\right)$.

Proof. Let $\pi$ be a partition of $[n]$ with $m$ blocks, let $M=M(\pi, m)$. We color the cells of $M$ red and green, where a cell in row $i$ and column $j$ is green if and only if the submatrix of $M$ formed by the intersection of the first $i-1$ rows and $j-1$ columns of $M$ contains $M(\tau, k)$. It is not difficult to see that for each green cell $(i, j)$ there is an occurrence of $M(\tau, k)$ which appears in the first $i-1$ rows and the first $j-1$ columns and which consists entirely of red cells. Thus, for any matrix $M^{\prime}$ obtained from $M$ by modifying the filling of $M$ 's green cells, the green cells of $M^{\prime}$ appear exactly at the same positions as the green cells of $M$.

Let $\Gamma$ be the diagram formed by the green cells of $M$, and let $G$ be the filling of $\Gamma$ by the values from $M$. Note that $\Gamma$ is an upside-down copy of a Ferrers shape. It is easy to see that the partition $\pi$ avoids $\sigma$ if and only if $G$ avoids $M\left(\bar{S}_{q}^{p}, 2\right)$, and $\pi$ avoids $\sigma^{\prime}$ if and only if $G$ avoids $M\left(\bar{S}_{0}^{p+q}, 2\right)$.

Let us now assume that $\pi$ is $\sigma$-avoiding. We now describe a procedure to transform $\pi$ into a $\sigma^{\prime}$-avoiding partition $\pi^{\prime}$ (see Figure 1). We first turn the filling $G$ and the diagram $\Gamma$ upside down, which transforms $\Gamma$ into a Ferrers shape $\bar{\Gamma}$, and it also transforms the $M\left(\bar{S}_{q}^{p}, 2\right)$-avoiding filling $G$ into an $M\left(S_{q}^{p}, 2\right)$-avoiding filling $\bar{G}$ of $\bar{\Gamma}$. Then we apply 
the bijection $f$ of Lemma 39 to $\bar{G}$, ignoring the zero columns of $\bar{G}$. We thus obtain a filling $\bar{G}^{\prime}=f(\bar{G})$ which avoids $M\left(S_{0}^{p+q}, 2\right)$. We turn this filling upside down, obtaining a $M\left(\bar{S}_{0}^{p+q}, 2\right)$-avoiding filling $G^{\prime}$ of $\Gamma$. We then fill the green cells of $M$ with the values of $G^{\prime}$ while the filling of the red cells remains the same. We thus obtain a matrix $M^{\prime}$. The matrix $M^{\prime}$ has exactly one 1-cell in each column, so there is a sequence $\pi^{\prime}$ over the alphabet $[m]$ such that $M^{\prime}=M\left(\pi^{\prime}, m\right)$.

By construction, the sequence $\pi^{\prime}$ has no subsequence order-isomorphic to $\sigma^{\prime}$. We now need to show that $\pi^{\prime}$ is a restricted-growth sequence. For this, we will use the preservation of the $t$-falling property. Let $c_{i}$ be the leftmost 1 -cell of the $i$-th row of $M$, let $c_{i}^{\prime}$ be the leftmost 1 -cell of the $i$-th row of $M^{\prime}$. We know that the cells $c_{1}, \ldots, c_{m}$ form an increasing chain, because $\pi$ was a restricted-growth sequence. We want to show that the cells $c_{1}^{\prime}, \ldots, c_{m}^{\prime}$ form an increasing chain as well. Let $s$ be the largest index such that the cell $c_{s}$ is red in $M$. We set $s=0$ if no such cell exists. Note that the cells $c_{1}, \ldots, c_{s}$ are red and the cells $c_{s+1}, \ldots, c_{m}$ are green in $M$. We have $c_{i}=c_{i}^{\prime}$ for every $i \leq s$. If $s>0$, we also see that all the green 1-cells of $M$ are in the columns to the right of $c_{s}$. This means that even in the matrix $M^{\prime}$ all the green 1-cells are to the right of $c_{s}$, because the empty columns of $G$ must remain empty in $G^{\prime}$. In particular, all the cells $c_{s+1}^{\prime}, \ldots, c_{m}^{\prime}$ appear to the right of $c_{s}^{\prime}$.

It remains to show that $c_{s+1}^{\prime}, \ldots, c_{m}^{\prime}$ form an increasing chain. We know that the cells $c_{s+1}, \ldots, c_{m}$ form an increasing chain in $M$ and in $G$. When $G$ is turned upside down, this chain becomes a decreasing chain $\overline{c_{s+1}}, \ldots, \overline{c_{m}}$ in $\bar{G}$. This chain shows that $\bar{G}$ is $(m-s)$-falling. By Lemma $39, \bar{G}^{\prime}$ must be $(m-s)$-falling as well, hence it contains a decreasing chain $\overline{c_{s+1}^{\prime}}, \ldots, \overline{c_{m}^{\prime}}$ in its bottom $m-s$ rows. This decreasing chain corresponds to an increasing chain $c_{s+1}^{\prime}, \ldots, c_{m}^{\prime}$ in $M^{\prime}$, showing that $\pi^{\prime}$ is a restricted-growth function, as claimed.

It is obvious that the above construction can be reversed, which shows that it is indeed a bijection between $P(n ; \sigma)$ and $P\left(n ; \sigma^{\prime}\right)$.

The following result is proved by a similar approach, but the argument is slightly more technical.

Theorem 42. Let $T$ be an arbitrary sequence over the alphabet $[k]$, let $p \geq 1$ and $q \geq 0$. The partition $\sigma=12 \cdots k\left(\bar{S}_{q}^{p}+k\right) T$ is equivalent to $\sigma^{\prime}=12 \cdots k\left(\bar{S}_{0}^{p+q}+k\right) T$.

Proof. Let $\pi$ be a partition of $[n]$ with $m$ blocks, let $M=M(\pi, m)$. As in the previous proof, we color the cells of $M$ red and green. A cell in row $i$ and column $j$ will be green if the submatrix of $M$ formed by rows $1, \ldots, i-1$ and columns $j+1, \ldots, n$ contains $M(T, k)$.

Let $\Gamma$ be the diagram formed by the green cells and $G$ its filling inherited from $M$. Let $r$ be the number of rows of $\Gamma$. The partition $\pi$ contains $\sigma$ if and only if $G$ contains $M\left(\bar{S}_{q}^{p}, 2\right)$. Note that the diagram $\Gamma$ is an upside-down copy of a left-justified stack polyomino.

We apply the same construction as in the previous proof. Let $\bar{G}$ be the upside down copy of $G$. The filling $\bar{G}$ is $r$-falling and it avoids $M\left(S_{q}^{p}, 2\right)$. We apply the mapping $f$ from Lemma 39 to transform $\bar{G}$ into an $r$-falling sparse filling $\bar{G}^{\prime}$. We then turn $\bar{G}^{\prime}$ upside down again and reinsert it into the green cells of the original matrix. This yields a matrix 


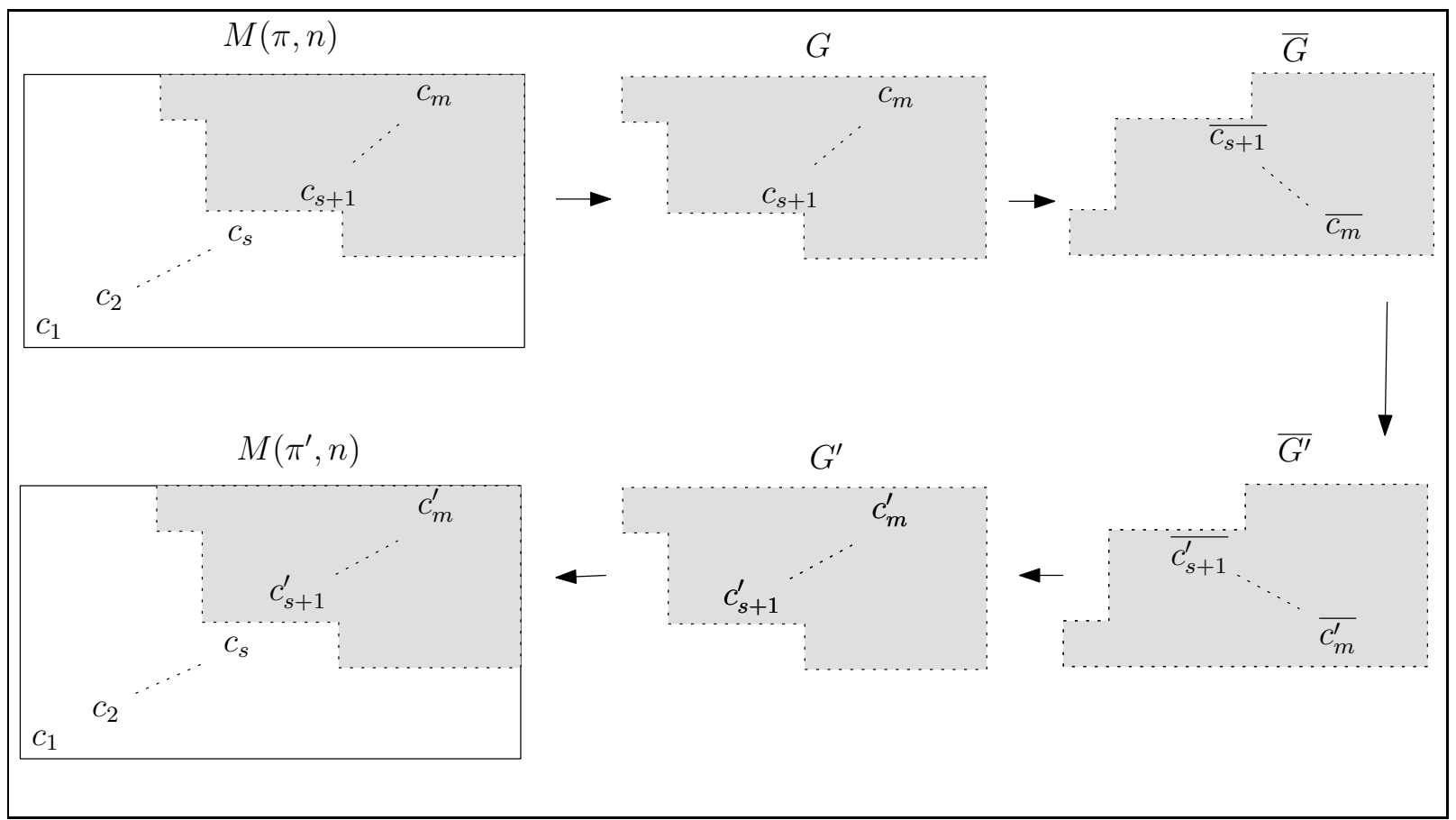

Figure 1: Illustration of the proof of Theorem 41.

$M^{\prime}$ with exactly one 1-cell in each column. Hence, there exists a sequence $\pi^{\prime}$, such that $M^{\prime}=M\left(\pi^{\prime}, m\right)$. The sequence $\pi^{\prime}$ has no subsequence order-isomorphic to $\sigma^{\prime}$.

We need to prove that $\pi^{\prime}$ is a restricted-growth sequence. Let $c_{i}$ be the leftmost 1-cell in row $i$ of $M$ and let $c_{i}^{\prime}$ be the leftmost 1-cell in row $i$ of $M^{\prime}$. To prove that $\pi^{\prime}$ is a partition, we want to show that $c_{1}^{\prime}, \ldots, c_{m}^{\prime}$ form an increasing chain in $M^{\prime}$.

Let us fix two row indices $i<j$. We claim that $c_{i}^{\prime}$ is left of $c_{j}^{\prime}$. If both $c_{i}^{\prime}$ and $c_{j}^{\prime}$ are green, then the claim follows from the preservation of the $r$-falling property. If both $c_{i}^{\prime}$ and $c_{j}^{\prime}$ are red, then $c_{i}^{\prime}=c_{i}$ and $c_{j}^{\prime}=c_{j}$. The claim then follows from the fact that $c_{1}, \ldots, c_{m}$ is an increasing chain. If $c_{j}^{\prime}$ is red and $c_{i}^{\prime}$ is green, the claim holds as well, because $c_{j}=c_{j}^{\prime}$, and all the green cells below row $j$ must appear to the left of the column of $c_{j}$.

Finally, assume that $c_{j}^{\prime}$ is green and $c_{i}^{\prime}$ is red. We have $c_{i}^{\prime}=c_{i}$. All the 1-cells of $G$ that are to the left of $c_{i}$ are also below row $i$. Let $x$ be the number of such 1-cells. Then $x$ is equal to the number of nonzero columns of $G$ that are to the left of $c_{i}$. Since the number of these nonzero columns is preserved by the mapping $f$, we see that $G^{\prime}$ also has $x$ 1-cells left of $c_{i}$.

Since $f$ preserves the number of 1 -cells in each row, both $G$ and $G^{\prime}$ have exactly $x$ 1-cells below row $i$. All the 1-cells of $G^{\prime}$ below row $i$ must appear to the left of $c_{i}$, and since there are only $x$ 1-cells of $G^{\prime}$ to the left of $c_{i}$, they must all appear below row $i$. Hence, all the green 1-cells above row $i$ (including the cell $c_{j}^{\prime}$ ) appear to the right of $c_{i}$. 


\subsection{Patterns equivalent to $12^{k} 13$}

Let $t$ be a nonnegative integer. In this subsection, we will deal with the following sets of patterns:

$$
\begin{aligned}
\Sigma_{t}^{+} & =\left\{12^{p+1} 12^{q} 32^{r}: p, q, r \geq 0, p+q+r=t\right\} \\
\Sigma_{t}^{-} & =\left\{12^{p+1} 32^{q} 12^{r}: p, q, r \geq 0, p+q+r=t\right\} \\
\Sigma_{t} & =\Sigma_{t}^{+} \cup \Sigma_{t}^{-}
\end{aligned}
$$

Our aim is to show that all the patterns in $\Sigma_{t}$ are equivalent. Throughout this subsection, we will assume that $t$ is arbitrary but fixed. We will write $\Sigma^{+}, \Sigma^{-}$and $\Sigma$ instead of $\Sigma_{t}^{+}, \Sigma_{t}^{-}$ and $\Sigma_{t}$, if there is no risk of ambiguity.

We will use the following definition.

Definition 43. Let $\sigma$ be a pattern over the alphabet $\{1,2,3\}$, let $\pi$ be a partition with $m$ blocks, and let $k \leq m$ be an integer. We say that $\pi$ contains $\sigma$ at level $k$, if there are symbols $\ell, h \in[m]$ such that $\ell<k<h$, and the partition $\pi$ contains a subsequence $S$ made of the symbols $\{\ell, k, h\}$ which is order-isomorphic to $\sigma$.

For example, the partition $\pi=1231323142221$ contains $\sigma=121223$ at level 3 , because $\pi$ contains the subsequence 131334, but $\pi$ avoids $\sigma$ at level 2 , because $\pi$ has no subsequence of the form $\ell 2 \ell 22 h$ with $\ell<2<h$.

Our plan is to show, for suitable pairs $\sigma, \sigma^{\prime} \in \Sigma$, that for every $k$ there is a bijection $f_{k}$ that maps the partitions avoiding $\sigma$ at level $k$ to the partitions avoiding $\sigma^{\prime}$ at level $k$, while preserving $\sigma^{\prime}$-avoidance at all levels $j<k$ and preserving $\sigma$-avoidance at all levels $j>k+1$. Composing the maps $f_{k}$ for $k=2, \ldots, n-1$, we will obtain a bijection between $P(n ; \sigma)$ and $P\left(n ; \sigma^{\prime}\right)$.

To do this we will need more definitions.

Definition 44. Consider a partition $\pi$, and fix a level $k \geq 2$. A symbol of $\pi$ is called $k$-low if it is smaller than $k$ and $k$-high if it is greater than $k$. A $k$-low cluster (or $k$-high cluster) is a maximal consecutive sequence of $k$-low symbols (or $k$-high symbols, respectively) in $\pi$. The $k$-landscape of $\pi$ is a word over the alphabet $\{\mathrm{L}, k, \mathrm{H}\}$ obtained from $\pi$ by replacing each $k$-low cluster with a single symbol L and each $k$-high cluster with a single symbol $\mathrm{H}$.

A word $w$ over the alphabet $\{\mathrm{L}, k, \mathrm{H}\}$ is called a $k$-landscape word if it satisfies the following conditions.

- The first symbol of $w$ is $\mathrm{L}$, the second symbol of $w$ is $k$.

- No two symbols L are consecutive in $w$, no two symbols $\mathrm{H}$ are consecutive in $w$.

Clearly, the landscape of a partition is a landscape word.

Two $k$-landscape words $w$ and $w^{\prime}$ are said to be compatible, if each of the three symbols $\{\mathrm{L}, k, \mathrm{H}\}$ has the same number of occurrences in $w$ as in $w^{\prime}$. 
We will often drop the prefix $k$ from these terms, if the value of $k$ is clear from the context.

To give an example, consider $\pi=1231323142221$ : it has five 3-low clusters, namely 12, 1, 2, 1 and 2221, it has one 3-high cluster 4, and its 3-landscape is L3L3L3LHL.

If $w$ and $w^{\prime}$ are two compatible $k$-landscape words, we have a natural bijection between partitions with landscape $w$ and partitions with landscape $w^{\prime}$. If $\pi$ has landscape $w$, we map $\pi$ to the partition $\pi^{\prime}$ of landscape $w^{\prime}$ which has the same $k$-low clusters and $k$-high clusters as $\pi$, and moreover, the $k$-low clusters appear in the same order in $\pi$ as in $\pi^{\prime}$, and also the $k$-high clusters appear in the same order in $\pi$ as in $\pi^{\prime}$. It is not difficult to check that these rules define a unique sequence $\pi^{\prime}$ and this sequence is indeed a partition. This provides a bijection between partitions of landscape $w$ and partitions of landscape $w^{\prime}$ which will be called the $k$-shuffle from $w$ to $w^{\prime}$.

The key property of shuffles is established by the next lemma.

Lemma 45. Let $w$ and $w^{\prime}$ be two compatible $k$-landscape words. Let $\pi$ be a partition with $k$-landscape $w$ and let $\pi^{\prime}$ be the partition obtained from $\pi$ by the shuffle from $w$ to $w^{\prime}$. Let $\sigma$ be a pattern from $\Sigma$, and let $j$ be an integer. The following holds.

1. If $\sigma$ does not end with the symbol 1 and $j>k$, then $\pi^{\prime}$ contains $\sigma$ at level $j$ if and only if $\pi$ contains $\sigma$ at level $j$.

2. If $\sigma$ does not end with the symbol 3 and $j<k$, then $\pi^{\prime}$ contains $\sigma$ at level $j$ if and only if $\pi$ contains $\sigma$ at level $j$.

Proof. We begin with the first claim of the lemma. Let $\sigma=12^{p+1} 32^{q} 12^{r}$ be an arbitrary pattern from $\Sigma^{-}$(the case $\sigma \in \Sigma^{+}$is analogous). By assumption, we have $r>0$. Assume that $\pi$ contains $\sigma$ at a level $j>k$. In particular, $\pi$ has a subsequence $S=\ell j^{p+1} h j^{q} \ell j^{r}$, with $\ell<j<h$.

If $k<\ell$, then all the symbols of $S$ are $k$-high. Since the shuffle preserves the relative order of high symbols, $\pi^{\prime}$ contains the subsequence $S$ as well. If $k \geq \ell$, then the shuffle preserves the relative order of the symbols $j$ and $h$, which are all high. Let $x$ and $y$ be the two symbols of $S$ directly adjacent to the second occurrence of $\ell$ in $S$ (if $q>0$, both these symbols are equal to $j$, otherwise one of them is $h$ and the other $j$ ). The two symbols are both high, but they must appear in different $k$-high clusters. After the shuffle, the two symbols $x$ and $y$ will again be in different clusters, separated by a non-high symbol $\ell^{\prime} \leq k$, and since the first occurrence of $\ell^{\prime}$ in $\pi^{\prime}$ precedes any occurrence of $j$, the partition $\pi^{\prime}$ will contain a subsequence $\ell^{\prime} j^{p+1} h j^{q} \ell^{\prime} j^{r}$, which is order-isomorphic to $\sigma$.

We see that the shuffle preserves the occurrence of $\sigma$ at level $j$. Since the inverse of the shuffle from $w$ to $w^{\prime}$ is the shuffle from $w^{\prime}$ to $w$, we see that the inverse of a shuffle preserves the occurrence of $\sigma$ at level $j$ as well.

The second claim of the lemma is proved by a similar argument. Assume that $\pi$ contains $\sigma$ at a level $j<k$. Thus, $\pi$ contains a subsequence $S$ over the alphabet $\{\ell<j<$ $h\}$, which is order-isomorphic to $\sigma$. If $h<k$, then the symbols of $S$ are low and hence preserved by the shuffle. If $h \geq k$, let $x$ and $y$ be the two symbols of $S$ adjacent to the symbol of $h$. Recall that $\sigma$ does not end with the symbol 3, so $x$ and $y$ are both well 
defined. The symbols $x$ and $y$ must appear in two distinct low clusters. After the shuffle is performed there will be a non-low symbol $h^{\prime}$ between $x$ and $y$. Hence, $\pi^{\prime}$ will contain a subsequence order isomorphic to $\sigma$.

We will use shuffles as basic building blocks for our bijection. The first example is the following lemma.

Lemma 46. For every $p, q, r \geq 0$, the pattern $\sigma=12^{p+1} 12^{q} 32^{r}$ is equivalent to the pattern $\sigma^{\prime}=12^{p+1} 32^{q} 12^{r}$.

Proof. Let us fix $p, q, r \geq 0$ and define $t=p+q+r$. For a given $k$, a partition $\pi$ of $[n]$ is called a $k$-hybrid if $\pi$ avoids $\sigma^{\prime}$ at every level $j<k$ and $\pi$ avoids $\sigma$ at every level $j \geq k$. We will show that for every $k \in\{2, \ldots, n-1\}$ there is a bijection $f_{k}$ between $k$-hybrids and $(k+1)$-hybrids. Since 2 -hybrids are precisely the $\sigma$-avoiding partitions of $[n]$ and $n$-hybrids are precisely the $\sigma^{\prime}$-avoiding partitions of $[n]$, this gives the required result.

Let us fix $k$. Note that a partition $\pi$ contains $\sigma$ at level $k$ if and only if its $k$-landscape $w$ contains a subsequence $k^{p+1} \mathrm{~L} k^{q} \mathrm{H} k^{r}$. Similarly, $\pi$ contains $\sigma^{\prime}$ at level $k$ if and only if $w$ contains a subsequence $k^{p+1} \mathrm{H} k^{q} \mathrm{~L} k^{r}$.

Let $\pi$ be a $k$-hybrid with landscape $w$. If $\pi$ has fewer than $t+1$ occurrences of $k$, then it is also a $(k+1)$-hybrid and we put $f_{k}(\pi)=\pi$. Otherwise, we write $w=x y z$, where $x$ is the shortest prefix of $w$ that has $p+1$ symbols $k$ and $z$ is the shortest suffix of $w$ that has $r$ symbols $k$. By assumption, $x$ and $z$ do not overlap (although they may be adjacent if $q=0$ ). Let $\bar{y}$ be the word obtained by reversing the order of the letters of $y$, define $w^{\prime}=x \bar{y} z$. Note that $w^{\prime}$ is a landscape word compatible with $w$, and that $w$ avoids $k^{p+1} \mathrm{~L} k^{q} \mathrm{H} k^{r}$ if and only if $w^{\prime}$ avoids $k^{p+1} \mathrm{H} k^{q} \mathrm{~L} k^{r}$. We apply to $\pi$ the shuffle from $w$ to $w^{\prime}$ which transforms it into a partition $\pi^{\prime}=f_{k}(\pi)$.

Lemma 45 implies that $\pi^{\prime}$ is a $(k+1)$-hybrid. Hence, $f_{k}$ is the required bijection.

Another result in the same spirit is the following lemma.

Lemma 47. For every $p, q, r \geq 0$, the pattern $\sigma=12^{p+2} 12^{q} 32^{r}$ is equivalent to the pattern $\sigma^{\prime}=12^{p+1} 12^{q} 32^{r+1}$.

Proof. We follow the same argument as in Lemma 46. As before, a $k$-hybrid is a partition that avoids $\sigma^{\prime}$ at every level $j<k$ and that avoids $\sigma$ at every level $j \geq k$. We will present a bijection $f_{k}$ between $k$-hybrids and $(k+1)$-hybrids. Note that $\pi$ avoids $\sigma$ at level $k$ if and only if its landscape $w$ avoids $k^{p+2} \mathrm{~L} k^{q} \mathrm{H} k^{r}$.

Fix a $k$-hybrid $\pi$ with a landscape $w$. If $\pi$ has fewer than $p+2+q+r$ occurrences of $k$, then it is also a $(k+1)$-hybrid and we define $f_{k}(\pi)=\pi$; otherwise, we write $w=x S y z$ where $x$ is the shortest prefix of $w$ that has $p+1$ occurrences of $k, z$ is the shortest suffix with $r$ occurrences of $k, S$ is the subword that starts just after the $(p+1)^{\text {th }}$ occurrence of $k$ and ends immediately after the $(p+2)^{\text {th }}$ occurrence of $k$. We define $w^{\prime}=x y \bar{S} z$, where $\bar{S}$ is the reversal of $S$.

Note that in the definition of $w^{\prime}$, we need to take $w^{\prime}=x y \bar{S} z$ instead of the seemingly more natural definition $w=x y S z$. This is because in general, the string $x y S z$ need not be a landscape word, since it may contain to consecutive occurrences of either L or 
H. Our definition guarantees that $w^{\prime}$ is a correct landscape word, and that $w^{\prime}$ avoids $k^{p+1} \mathrm{~L} k^{q} \mathrm{H} k^{r+1}$ if and only if $w$ avoids $k^{p+2} \mathrm{~L} k^{q} \mathrm{H} k^{r}$ (which is if and only if $y$ avoids $\mathrm{L} k^{q} \mathrm{H}$ ).

The rest of the argument is the same as in the previous lemma.

We may now state and prove the main result of this subsection.

Theorem 48. For every $t$, the patterns in the set $\Sigma_{t}$ are equivalent.

Proof. By Corollary 40, we already know that for any $p, q \geq 0$, the pattern $12^{p+1} 12^{q} 3$ is equivalent to the pattern $12^{p+q+1} 13$. This, together with the two previous lemmas gives the required result.

\subsection{More 'landscape' patterns}

We will show that with a little bit of additional effort, the previous argument involving landscapes can be adapted to prove, for every $p, q \geq 0$, the following equivalences:

- $1232^{p} 412^{q} \sim 1232^{p} 42^{q} 1$

- $1232^{p} 142^{q} \sim 12312^{p} 42^{q}$

- $123^{p+1} 143^{q} \sim 123^{p+1} 13^{q} 4$

- $123^{p+1} 413^{q} \sim 12343^{p} 13^{q}$

Throughout this subsection, we will say that $\tau$ is a 1-2-4 pattern if $\tau$ has the form $123 S$ where $S$ is a sequence that has exactly one occurrence of the symbol 1, exactly one occurrence of the symbol 4 , and all its remaining symbols are equal to 2 , where 4 is neither the first nor the last symbol of $S$. Similarly, a 1-3-4 pattern is a pattern of the form $123 S$ where $S$ has one occurrence of 1 and of 4 , and all its other symbols are equal to 3 , where 1 is not the last symbol of $S$.

We decided to exclude the patterns of the form $1232^{p} 12^{q} 4,12342^{p} 12^{q}$ and $1233^{p} 43^{q} 1$ from the set of 1-2-4 and 1-3-4 patterns defined above, because some of the arguments we will need in the following discussion (namely in Lemma 49) would become more complicated if these special types of patterns were allowed. We need not be too concerned about this constraint, because we have already dealt with the patterns of the three excluded types in Corollary 40 and Theorem 42. From Corollary 40, we obtain the equivalences $1232^{p} 12^{q} 4 \sim 1232^{p+q} 14$ and $12342^{p} 12^{q} \sim 12342^{p+q} 1$, while from Theorem 42 , we obtain $1233^{p} 43^{q} 1 \sim 1233^{p+q} 41$.

For our arguments, we need to extend some of the terminology of the previous subsection to cover the new family of patterns. Let $\tau$ be a 1-2-4 pattern, $k$ be a natural number, and $\pi$ be a partition. We say that $\pi$ contains $\tau$ at level $k$, if $\pi$ has a subsequence $T$ order-isomorphic to $\tau$ such that the occurrences of the symbol 2 in $\tau$ correspond to the occurrences of the symbol $k$ in $T$. Similarly, if $\tau$ is a 1-3-4 pattern, we say that a partition $\pi$ contains $\tau$ at level $k$ if $\pi$ has a subsequence $T$ order-isomorphic to $\tau$ with the symbol $k$ in $T$ corresponding to the symbol 3 in $\tau$. 
Our aim is to prove an analogue of Lemma 45 for 1-2-4 and 1-3-4 patterns. Unfortunately, general $k$-shuffles may behave badly with respect to the avoidance of these patterns. However, we will define special types of $k$-shuffles that have the properties we need. We first introduce some new definitions.

Let $w$ be a $k$-landscape word. We say that two occurrences of the symbol $\mathrm{H}$ in $w$ are separated if there is at least one occurrence of L between them. Similarly, two symbols $\mathrm{L}$ are separated if there is at least one $\mathrm{H}$ between them. As an example, consider the $k$-landscape word $w=\mathrm{L} k \mathrm{~L} k \mathrm{H} k k \mathrm{HL} k \mathrm{H}$. In $w$, neither the first two occurrences of $\mathrm{L}$ nor the first two occurrences of $\mathrm{H}$ are separated, while the second and third occurrence of $\mathrm{H}$, as well as the second and third occurrence of $\mathrm{L}$ are separated. We also say that two clusters of a partition are separated if the corresponding symbols of the landscape word are separated.

Let $w$ and $w^{\prime}$ be two $k$-landscape words. We say that $w$ and $w^{\prime}$ are $H$-compatible if they are compatible, and if for any $i, j$, the $i$-th and $j$-th occurrence of $\mathrm{H}$ in $w$ are separated if and only if the $i$-th and $j$-th occurrence of $\mathrm{H}$ in $w^{\prime}$ are separated. An L-compatible pair of words is defined analogously.

For example, the two compatible words $w=\mathrm{L} k \mathrm{H} k k \mathrm{HL}$ and $w^{\prime}=\mathrm{L} k \mathrm{H} k \mathrm{LH} k$ are Lcompatible (since the two occurrences of $\mathrm{L}$ are separated in both words) but they are not $\mathrm{H}$-compatible (the two symbols $\mathrm{H}$ are not separated in $w$ but they are separated in $w^{\prime}$ ).

The following lemma explains the relevance of these concepts.

Lemma 49. Let $k$ be an integer. The following holds.

(1) Let $w$ and $w^{\prime}$ be two L-compatible k-landscape words, and let $\tau$ be a 1-2-4 pattern. Let $\pi$ be an arbitrary partition, and let $\pi^{\prime}$ be the partition obtained from $\pi$ by the $k$-shuffle from $w$ to $w^{\prime}$. For every $j<k, \pi$ contains $\tau$ at level $j$ if and only if $\pi^{\prime}$ contains $\tau$ at level $j$. Moreover, if the last symbol of $\tau$ is equal to 2 , then the previous equivalence also holds for every $j>k$.

(2) Let $w$ and $w^{\prime}$ be two H-compatible $k$-landscape words, and let $\tau$ be a 1-3-4 pattern. Let $\pi$ be an arbitrary partition, and let $\pi^{\prime}$ be the partition obtained from $\pi$ by the $k$-shuffle from $w$ to $w^{\prime}$. For every $j>k, \pi$ contains $\tau$ at level $j$ if and only if $\pi^{\prime}$ contains $\tau$ at level $j$. Moreover, if the last symbol of $\tau$ is equal to 3 , then the previous equivalence also holds for every $j<k$.

Proof. We first prove (1). Assume that $\pi$ contains a 1-2-4 pattern $\tau$ at level $j$. If $j>k$, we may use the same argument as in the proof of the first part of Lemma 45 to see that the occurrence of $\tau$ is preserved by the shuffle as long as $\tau$ does not end with a 1 .

Assume now that $j<k$. Let us write $\tau=1232^{p} 42^{q} 12^{r}$ (the case when $\tau$ has the form $1232^{p} 12^{q} 42^{r}$ is analogous). By assumption, $\pi$ contains a subsequence $T$ order-isomorphic to $\tau$, with the symbol 2 of $\tau$ corresponding to the symbol $j$ in $T$. We label from left-toright the $1+p+q+r$ occurrences of $j$ in $T$ by $j_{0}, j_{1}, \cdots, j_{p+q+r}$. Let $a<b<c$ denote the symbols of $T$ that correspond respectively to the symbols 1,3 and 4 in $\tau$; we label the two occurrences of $a$ in $T$ by $a_{0}$ and $a_{1}$. With this notation, we may write $T$ as follows:

$$
T=a_{0} j_{0} b j_{1} \cdots j_{p} c j_{p+1} \cdots j_{p+q} a_{1} j_{p+q+1} \cdots j_{p+q+r} .
$$


We distinguish several cases, based on the relative order of $b, c$ and $k$. If $c<k$, then all the symbols of $T$ are $k$-low and their relative position is preserved by the shuffle, which means that $T$ is also a subsequence of $\pi^{\prime}$.

If $c \geq k$ and $b<k$, then the symbols $a<j<b$ are $k$-low. Let $x$ and $y$ be the two symbols adjacent to $c$ in $T$. Typically $x=j_{p}$ and $y=j_{p+1}$, unless $q$ is zero, in which case $y=a_{1}$. Recall that $c$ cannot directly follow $b$ and it cannot be the last element of $T$ by the definition of 1-2-4 pattern. The elements $x$ and $y$ are low and they appear in two distinct low clusters. After the shuffle, the occurrences of $a, b$ and $j$ in $T$ have the same relative order, and the elements $x$ and $y$ still belong to different clusters. Thus, $\pi^{\prime}$ contains a symbol greater than $b$ between $x$ and $y$. This shows that $\pi^{\prime}$ has a subsequence order-isomorphic to $\tau$.

It remains to consider the most complicated case, when $c>k$ and $b \geq k$. This is when we first use the L-compatibility assumption. Let $x$ and $y$ be the two symbols adjacent to $c$ in $T$. By the definition of $1-2-4$ patterns, $x$ and $y$ are both $k$-low. Since $b$ is not $k$-low and $c$ is high, the partition $\pi$ has the following properties.

1. The symbol $j_{1}$ does not belong to the leftmost low cluster.

2. The two symbols $x$ and $y$ belong to two separated low clusters.

The two properties are preserved by the shuffle. In particular, in $\pi^{\prime}$, the symbol $j_{1}$ does not belong to the leftmost low cluster, which means that there is at least one non-low symbol appearing in $\pi^{\prime}$ before $j_{1}$. Since $\pi^{\prime}$ is a partition in its canonical sequential form, this implies that all the symbols $1,2, \cdots, k$ appear in $\pi^{\prime}$ in this order before $j_{1}$. Let $a^{\prime}, j^{\prime}$ and $k^{\prime}$ denote respectively the leftmost occurrences of $a, j$ and $k$ in $\pi^{\prime}$. We also know, from the L-compatibility of $w$ and $w^{\prime}$, that in $\pi^{\prime}$ the two symbols $x$ and $y$ appear in distinct and separated low clusters. In particular, $\pi^{\prime}$ contains a $k$-high symbol $c^{\prime}$ between $x$ and $y$. Putting it all together, we see that $\pi^{\prime}$ contains the subsequence

$$
T^{\prime}=a^{\prime} j^{\prime} k^{\prime} j_{1} \cdots j_{p} c^{\prime} j_{p+1} \cdots j_{p+q} a_{1} j_{p+q+1} \cdots j_{p+q+r},
$$

which is order isomorphic to $\tau$.

Thus, $\pi$ contains a 1-2-4 pattern $\tau$ at level $j$, if and only if $\pi^{\prime}$ contains $\tau$ at level $j$. This completes the proof of (1).

Claim (2) is proved by a similar argument. Let $\tau$ be a 1-3-4 pattern of the form $123^{p+1} 13^{q} 43^{r}$ (the case when $\tau=123^{p+1} 43^{q} 13^{r}$ is analogous and easier). Assume that $\pi$ contains $\tau$ at level $j$, represented by a sequence $T$ of the form

$$
T=a_{0} b j_{0} j_{1} \cdots j_{p} a_{1} j_{p+1} \cdots j_{p+q} c j_{p+q+1} \cdots j_{p+q+r}
$$

with $a<b<j<c$.

If $j<k$, we apply the same argument as in the proof of the second claim of Lemma 45 to prove that if $\tau$ does not end with 4 , then the occurrence of $\tau$ is preserved by the shuffle.

Next, we assume that $j>k$ and we distinguish several cases based on the relative order of $a, b$ and $k$. 
If $a>k$, then all the symbols of $T$ are $k$-high and their order is preserved by the shuffle.

If $a \leq k$, and $b>k$, we let $x$ and $y$ denote the two symbols adjacent to $a_{1}$ in $T$, and we observe that $\pi^{\prime}$ has a non-high element $a^{\prime}$ between $x$ and $y$. The first occurrence of $a^{\prime}$ in $\pi^{\prime}$ must appear to the left of any $k$-high symbol, hence $\pi^{\prime}$ contains a subsequence $a^{\prime} b j^{p+1} a^{\prime} j^{q} c j^{r}$ order-isomorphic to $\tau$.

If $a<k$ and $b \leq k$, we define $x$ and $y$ as in the previous paragraph. This time, $x$ and $y$ belong to two separated high clusters, so $\pi^{\prime}$ has a $k$-low element $a^{\prime}$ between $x$ and $y$, and in particular, $\pi^{\prime}$ contains the subsequence $a^{\prime} k j^{p+1} a^{\prime} j^{q} c j^{r}$.

With the help of Lemma 49, we may prove all the equivalence relations announced at the beginning of this section. We split the proofs into four lemmas and then summarize the results in a theorem.

Lemma 50. Let $p, q \geq 1$. The pattern $\tau=1232^{p} 412^{q}$ is equivalent to $\tau^{\prime}=1232^{p} 42^{q} 1$.

Proof. For an integer $k$ we say that a partition $\pi$ is a $k$-hybrid if $\pi$ avoids $\tau^{\prime}$ at level $j$ for every $j<k$ and it avoids $\tau$ at level $j$ for every $j \geq k$. To prove the claim, it is enough to establish a bijection $f_{k}$ between $k$-hybrids and $(k+1)$-hybrids.

We say that a $k$-high cluster of $\pi$ is extra-high if it contains a symbol greater than $k+1$. We claim that $\pi$ contains $\tau$ at level $k$ if and only if by scanning the $k$-landscape $w$ of $\pi$ from left to right we may find (not necessarily consecutively) the leftmost high cluster, followed by $p$ occurrences of the symbol $k$, followed by an extra-high cluster, followed by a low cluster, followed by $q$ occurrences of $k$. To see this, it suffices to notice that the leftmost high cluster contains the symbol $k+1$, and to the left of this cluster we may always find all the symbols $12 \cdots k$ in increasing order.

By a similar argument, we see that $\pi$ contains $\tau^{\prime}$ at level $k$ if and only if it contains, leftto-right, the leftmost high-cluster, $p$ occurrences of $k$, an extra-high cluster, $q$ occurrences of $k$ and a low cluster.

Now assume that $\pi$ is a $k$-hybrid partition. Let $\mathrm{H}^{\prime}$ be the leftmost extra-high cluster of $\pi$ such that between $\mathrm{H}^{\prime}$ and the leftmost high cluster of $\pi$ there are at least $p$ occurrences of $k$. If no such cluster exists, or if $\pi$ has fewer than $q$ symbols equal to $k$ to the right of $\mathrm{H}^{\prime}$, then $\pi$ avoids both $\tau$ and $\tau^{\prime}$ at level $k$, and we define $f_{k}(\pi)=\pi$.

Otherwise, let $w$ be the $k$-landscape of $\pi$. We will decompose $w$ as

$$
w=x \mathrm{H}^{\prime} y k_{q} S_{1} k_{q-1} S_{2} \cdots k_{1} S_{q},
$$

where $\mathrm{H}^{\prime}$ represents the extra-high cluster defined above, and $k_{i}$ represents the $i$-th symbol $k$ in $\pi$, counted from the right. The symbols $x, y$ and $S_{1}, \ldots, S_{q}$ above refer to the corresponding subwords of $w$ appearing between these symbols.

By construction, none of the $S_{i}$ 's contains the symbol $k$, so each of them is an alternating sequence over the alphabet $\{\mathrm{L}, \mathrm{H}\}$, possibly empty. Since $\pi$ avoids $\tau$ at level $k$, the subword $y$ does not contain the symbol L.

We decompose $S_{1}$ into two parts $S_{1}=H^{*} S_{1}^{-}$in the following way. If the first letter of $S_{1}$ is $\mathrm{H}$, then we put $H^{*}=\mathrm{H}$ and $S_{1}^{-}$is equal to $S_{1}$ with the first letter removed. If $S_{1}$ does not start with $\mathrm{H}$, then $H^{*}$ is the empty string and $S_{1}^{-}=S_{1}$. 
Now, we define the word $w^{\prime}$ by

$$
w^{\prime}=x \mathrm{H}^{\prime} S_{1}^{-} k_{1} S_{2} k_{2} S_{3} k_{3} \cdots k_{q-1} S_{q} k_{q} H^{*} y .
$$

It is not difficult to check that $w^{\prime}$ is a landscape word (note that neither $y$ nor $S_{1}^{-}$can start with the symbol $\mathrm{H}$ ), and that $w^{\prime}$ is L-compatible with $w$ (recall that $y$ contains no L).

Let $\pi^{\prime}$ be the partition obtained from $\pi$ by the shuffle from $w$ to $w^{\prime}$. Note that the prefix of $\pi$ through the cluster $\mathrm{H}^{\prime}$ is not affected by the shuffle, because the words $w$ and $w^{\prime}$ share the same prefix up to the symbol $\mathrm{H}^{\prime}$. In particular, the shuffle preserves the property that $\mathrm{H}^{\prime}$ is the leftmost extra-high cluster with at least $p$ symbols $k$ between $\mathrm{H}^{\prime}$ and the leftmost high cluster of $\pi^{\prime}$. It is routine to check that $\pi^{\prime}$ avoids $\tau^{\prime}$ at level $k$. By Lemma $49, \pi^{\prime}$ is a $(k+1)$-hybrid partition. It is easy to see that for any given $(k+1)$-hybrid partition $\pi^{\prime}$, we may uniquely invert the procedure above and obtain a $k$-hybrid partition $\pi$.

Defining $f_{k}(\pi)=\pi^{\prime}$, we obtain the required bijection between $k$-hybrids and $(k+1)$ hybrids.

The proofs of the following three lemmas follow the same basic argument as the proof of Lemma 50 above. The only difference is in the decompositions of the corresponding landscape words $w$ and $w^{\prime}$. We omit repeating the common parts of the arguments and concentrate on pointing out the differences.

Lemma 51. Let $p, q \geq 1$. The pattern $\tau=1232^{p} 142^{q}$ is equivalent to $\tau^{\prime}=12312^{p} 42^{q}$.

Proof. A partition $\pi$ contains $\tau$ at level $k$ if and only if it contains, from left to right, the leftmost high cluster, $p$ copies of $k$, a low cluster, an extra-high cluster, and $q$ copies of $k$. Similar characterization applies to $\tau^{\prime}$.

Let $\mathrm{H}_{1}$ denote the leftmost high cluster of $\pi$, let $\mathrm{H}^{\prime}$ denote the rightmost extra-high cluster of $\pi$ that has the property that there are at least $q$ occurrences of $k$ to the right of $\mathrm{H}^{\prime}$. If $\mathrm{H}^{\prime}$ does not exist, or if there are fewer than $p$ occurrences of $k$ between $\mathrm{H}_{1}$ and $\mathrm{H}^{\prime}$, then $\pi$ contains neither $\tau$ nor $\tau^{\prime}$ at level $k$ and we put $f_{k}(\pi)=\pi$. Otherwise, let $w$ be the landscape of $\pi$, and let us write

$$
w=x \mathrm{H}_{1} S_{1} k_{1} S_{2} k_{2} \cdots S_{p} k_{p} y \mathrm{H}^{\prime} z
$$

where none of the $S_{i}$ contains $k$, and $y$ avoids L. Define $S_{p}^{-}$and $H^{*}$ by writing $S_{p}=S_{p}^{-} H^{*}$ where $S_{p}^{-}$does not end with the letter $\mathrm{H}$ and $H^{*}$ is equal either to $\mathrm{H}$ or to the empty string, depending on whether $S_{p}$ ends with $\mathrm{H}$ or not.

Now we write

$$
w^{\prime}=x \mathrm{H}_{1} \bar{y} k_{1} H^{*} S_{1} k_{2} S_{2} \cdots k_{p} S_{p}^{-} \mathrm{H}^{\prime} z
$$

where $\bar{y}$ is the reversal of $y$. The rest of the proof is analogous to Lemma 50.

We now apply the same arguments to 1-3-4 patterns.

Lemma 52. For any $p \geq 0$ and $q \geq 1$, the pattern $\tau=123^{p+1} 13^{q} 4$ is equivalent to the pattern $\tau^{\prime}=123^{p+1} 143^{q}$. 
Proof. As usual, a $k$-hybrid is a partition that avoids $\tau$ at every level $j \geq k$ and that avoids $\tau^{\prime}$ at every level below $k$.

Let us say that a $k$-cluster of a partition $\pi$ is extra-low if it contains a symbol smaller than $k-1$. A partition contains $\tau$ at level $k$ if and only if it has $p+1$ occurrences of $k$ followed by an extra-low cluster, followed $q$ occurrences of $k$, followed by a high cluster. Similarly, a partition contains $\tau^{\prime}$ at level $k$ if and only if it has $p+1$ copies of $k$, followed by an extra-low cluster, followed by a high cluster, followed by $q$ copies of $k$.

Assume $\pi$ is a $k$-hybrid partition. Let $\mathrm{L}^{\prime}$ denote the leftmost extra-low cluster of $\pi$ that has at least $p+1$ copies of $k$ to its left. If $\mathrm{L}^{\prime}$ does not exist, or if it has fewer than $q$ copies of $k$ to its right, we put $f_{k}(\pi)=\pi$. Otherwise, we decompose the landscape word $w$ of $\pi$ as

$$
w=x \mathrm{~L}^{\prime} S_{1} k_{1} S_{2} k_{2} \cdots S_{q-1} k_{q-1} S_{q} k_{q} y,
$$

where the $S_{i}$ do not contain $k$. By assumption, $y$ avoids $H$. Next, we write $y=L^{*} y^{-}$ where $L^{*}$ is an empty string or a single symbol $\mathrm{L}$, and $y^{-}$does not start with $\mathrm{L}$. We define $w^{\prime}$ by

$$
w^{\prime}=x \mathrm{~L}^{\prime} y^{-} k_{1} L^{*} S_{1} k_{2} \cdots S_{q-1} k_{q} S_{q} .
$$

The words $w$ and $w^{\prime}$ are H-compatible. We define the bijection between $k$-hybrids and $(k+1)$-hybrids in the usual way.

Lemma 53. For every $p \geq 0$ and $q \geq 1$, the pattern $\tau=123^{p+1} 413^{q}$ is equivalent to the pattern $\tau^{\prime}=12343^{p} 13^{q}$.

Proof. As before, take $\pi$ to be a $k$-hybrid partition. Let $\mathrm{L}^{\prime}$ be the rightmost extra-low cluster that has at least $q$ copies of $k$ to its right. If $\mathrm{L}^{\prime}$ has at least $p+1$ copies of $k$ to its left, we decompose the landscape $w$ of $\pi$ as

$$
w=\mathrm{L} k_{1} S_{1} k_{2} S_{2} \cdots k_{p} S_{p} k_{p+1} y \mathrm{~L}^{\prime} z .
$$

Next, we write $S_{p}=S_{p}^{-} L^{*}$ with the usual meaning and define

$$
w^{\prime}=\mathrm{L} k_{1} L^{*} \bar{y} k_{2} S_{1} k_{3} S_{2} \cdots S_{p-1} k_{p+1} S_{p}^{-} \mathrm{L}^{\prime} z .
$$

The rest is the same as before.

We summarize our results.

Theorem 54. For every $p, q \geq 0$, we have the following equivalences:
1. $1232^{p} 412^{q} \sim 1232^{p} 42^{q} 1$
2. $1232^{p} 142^{q} \sim 12312^{p} 42^{q}$
3. $123^{p+1} 143^{q} \sim 123^{p+1} 13^{q} 4$
4. $123^{p+1} 413^{q} \sim 12343^{p} 13^{q}$ 
Proof. If $p$ and $q$ are both positive, the results follow directly from the four preceding lemmas.

If $p=0$, the second and the fourth claim are trivial, the first one is a special case of Corollary 40, and the third is covered by Lemma 52.

If $q=0$, the first and the third claim are trivial, the second is a special case of Corollary 40, and the fourth follows from Theorem 42.

\section{The patterns of size four}

\subsection{Enumeration of 1123-avoiding partitions}

To complete the classification of the equivalence classes of the patterns of length four, we need to prove the equivalence $1212 \sim 1123$. Unlike in the previous arguments, we do not present a direct bijection between pattern-avoiding classes, but rather we prove that $p(n ; 1123)$ is equal to the $n$-th Catalan number. Since it is well known that noncrossing partitions are enumerated by the Catalan numbers (see, e.g., [16]), this will yield the desired equivalence.

As we said before, our aim is to show that the 1123 -avoiding partitions of $[n]$ are enumerated by the $n$-th Catalan number, i.e., $p(n ; 1123)=\frac{1}{n+1}\left(\begin{array}{c}2 n \\ n\end{array}\right)$.

We achieve this by proving that $p(n ; 1123)$ is equal to the number of Dyck paths of semilength $n$. A Dyck path of semilength $n$ is a nonnegative path on the two-dimensional integer lattice from $(0,0)$ to $(2 n, 0)$ composed of up-steps connecting $(x, y)$ to $(x+1, y+1)$ and down-steps connecting $(x, y)$ to $(x+1, y-1)$. It is well known that these paths are enumerated by Catalan numbers. Let $D(n, k)$ be the set of Dyck paths of semilength $n$ whose last up-step is followed by exactly $k$ down-steps. Let $d(n, k)$ be the cardinality of $D(n, k)$.

Lemma 55. The numbers $d(n, k)$ are determined by the following set of recurrences:

$$
\begin{aligned}
& d(1,1)=1 \\
& d(n, k)=0 \quad \text { if } \quad k<1 \quad \text { or } \quad k>n \\
& d(n, k)=\sum_{j=k-1}^{n-1} d(n-1, j) \quad \text { for } \quad n \geq 2, n \geq k \geq 1
\end{aligned}
$$

Proof. Only the third recurrence is nontrivial. We prove it by presenting a bijection between $D(n, k)$ and the disjoint union $\bigcup_{j=k-1}^{n-1} D(n-1, j)$. Assume that $k$ and $n$ are fixed, with $n \geq 2$ and $k \leq n$. Take a Dyck path $P \in D(n, k)$. By erasing the last up-step and the last down-step of $D$, we get a Dyck path $P^{\prime} \in D(n-1, j)$, where $j \geq k-1$. Conversely, given a Dyck path $P^{\prime} \in D(n-1, j)$ with $j \geq k-1$, we insert a down-step at the end of $D^{\prime}$, and then insert an up-step into the resulting path immediately before its last $k$ down-steps. This inverts the mapping above. 
We now focus on 1123-avoiding partitions. First of all, we will present a correspondence between 1123-avoiding partitions and 123-avoiding sequences. Before we state the correspondence, we need some more definitions.

Definition 56. A 123-avoiding sequence is a sequence $s_{1}, s_{2}, \ldots, s_{\ell}$ of positive integers, such that there are no three indices $i<j<k$ that would satisfy $s_{i}<s_{j}<s_{k}$. We define the rank of a sequence to be equal to $\ell+m-1$, where $\ell$ is the length of the sequence and $m=\max \left\{s_{i}, i=1, \ldots, \ell\right\}$ is the largest element of the sequence.

For example, there are five 123-avoiding sequences of rank $3:(1,1,1),(1,2),(2,1),(2,2)$, and (3). There are fourteen 123-avoiding sequences of rank 4: $(1,1,1,1),(1,1,2),(1,2,1)$, $(1,2,2),(2,1,1),(2,1,2),(2,2,1),(2,2,2),(1,3),(2,3),(3,1),(3,2),(3,3)$, and $(4)$.

Claim 57. A 1123-avoiding partition $\pi$ of $[n]$ with $m$ blocks has the following form:

$$
\pi=123 \cdots(m-2)(m-1) S
$$

where $S$ is a 123-avoiding sequence of rank $n$, with maximum element $m$. Conversely, If $S$ is any 123-avoiding sequence of rank $n$ with maximum element $m$ then $\pi$ defined by the formula (8) is a canonical sequence of a 1123-avoiding partition of $[n]$.

In particular, the number of 123-avoiding sequences of rank $n$ with last element $k$ is equal to the number of 1123-avoiding partitions of size $n$ with last element $k$.

Proof. Let $\pi=\pi_{1} \cdots \pi_{n}$ be a 1123-avoiding partition with $m$ blocks. Observe that for every $i \in[m-1]$, the symbol $\pi_{i}$ is equal to $i$, otherwise $\pi$ would contain the forbidden pattern. It follows that $\pi$ can be decomposed as $\pi=123 \cdots(m-2)(m-1) S$, where the sequence $S$ has length $l=n-m+1$ and maximum element equal to $m$. In particular, $S$ has rank $n$ and its last element is equal to $k$.

We now check that $S$ is 123 -avoiding. If $S$ contained a subsequence $x y z$ for $x<y<z$ then the original partition would contain a subsequence $x x y z$, which is forbidden. It follows that $S$ obtained from a 1123-avoiding partition $\pi$ has all the required properties.

Conversely, if $S$ is a 123-avoiding sequence of rank $n$ and maximum element $m$, then it is routine to verify that $\pi=12 \cdots(m-1) S$ is a 1123 -avoiding partition of size $n$ with $m$ blocks. Clearly, the last element of $\pi$ is equal to the last element of $S$.

Let $T(n, k)$ be the set of 123 -avoiding sequences of rank $n$ with last element equal to $k$. Let $t(n, k)$ be the cardinality of $T(n, k)$. By the previous claim, $t(n, k)$ is equal to the number of 1123-avoiding partitions of size $n$ with last element equal to $k$. To show that 1123-avoiding partitions of size $n$ have the same enumeration as Dyck paths of semilength $n$, it suffices to show that $d(n, k)=t(n, k)$ for each $n, k$. To show this, we will prove that $t(n, k)$ is determined by the same set of recurrences as $d(n, k)$.

Claim 58. The numbers $t(n, k)$ satisfy the following set of recurrences:

$$
\begin{aligned}
t(1,1) & =1 \\
t(n, k) & =0 \quad \text { if } \quad k<1 \quad \text { or } \quad k>n \\
t(n, k) & =\sum_{i=k-1}^{n-1} t(n-1, i) \quad \text { for } \quad n \geq 2, n \geq k \geq 1
\end{aligned}
$$


Proof. Only the recurrence (11) is nontrivial. Let us fix $n \geq 2$ and $k \leq n$. To prove the recurrence, we need a bijection from $T(n, k)$ to $\cup_{i=k-1}^{n-1} T(n-1, i)$.

Let us first consider the case $k=1$. A sequence $S \in T(n, 1)$ can be transformed into a sequence $S^{\prime} \in \cup_{i=0}^{n-1} T(n-1, i)$, by simply erasing the last element of $S$. This provides a bijection between $T(n, 1)$ and $\cup_{i=0}^{n-1} T(n-1, i)$.

In the rest of the proof, we deal with the case $k>1$. Let $S \in T(n, k)$ be a 123 -avoiding sequence of length $\ell$. The sequence $S$ can be uniquely expressed as $S=S_{0} 1^{b} k$, where $S_{0}$ is the (possibly empty) longest proper prefix of $S$ whose last element is different from 1. If $S_{0}$ is nonempty, let $j$ be the last element of $S_{0}$.

Let us decompose $T(n, k)$ into a disjoint union of two sets $T_{1}$ and $T_{2}$ defined by

$$
\begin{aligned}
& T_{1}=\left\{S \in T(n, k): \quad S_{0} \text { is nonempty, and } j \geq k\right\} \\
& T_{2}=\left\{S \in T(n, k): \quad S_{0} \text { is empty, or } j<k\right\} .
\end{aligned}
$$

Note that if $S \in T_{2}$ and $S_{0}$ is nonempty, then all the elements of $S_{0}$ are greater than or equal to $j$. Indeed, if $S_{0}$ contained an element $i$ smaller than $j$, then $S$ would contain a subsequence $i j k$, which would create a copy of 123 in $S$.

Let $S^{\prime}$ be a sequence from $\cup_{j=k-1}^{n-1} T(n-1, j)$. $S^{\prime}$ may be uniquely expressed as $S^{\prime}=$ $S_{0}^{\prime}(k-1)^{c}$, where $c \geq 0$ and $S_{0}^{\prime}$ is the (possibly empty) longest prefix of $S^{\prime}$ whose last element is different from $k-1$. Note that if the last element of $S^{\prime}$ is greater than $k-1$ then $S^{\prime}=S_{0}^{\prime}$. If $S_{0}^{\prime}$ is nonempty, let $j^{\prime}$ be the last element of $S_{0}^{\prime}$.

We decompose $\cup_{j=k-1}^{n-1} T(n-1, j)$ into a disjoint union of two sets $T_{1}^{\prime}$ and $T_{2}^{\prime}$, where

$$
\begin{aligned}
& T_{1}^{\prime}=\left\{S^{\prime} \in \cup_{i=k-1}^{n-1} T(n-1, i): \quad S_{0}^{\prime} \text { is nonempty, and } j^{\prime} \geq k\right\} \\
& T_{2}^{\prime}=\left\{S^{\prime} \in \cup_{i=k-1}^{n-1} T(n-1, i): \quad S_{0}^{\prime} \text { is empty, or } j^{\prime}<k-1\right\} .
\end{aligned}
$$

Since $j^{\prime}$ is never equal to $k-1$, the two sets $T_{1}^{\prime}$ and $T_{2}^{\prime}$ form a disjoint partition of $\cup_{i=k-1}^{n-1} T(n-1, i)$. Note that $T_{2}^{\prime}$ is in fact a subset of $T(n-1, k-1)$.

To prove the claim, it suffices to give a bijection $f_{1}$ between $T_{1}$ and $T_{1}^{\prime}$, and a bijection $f_{2}$ between $T_{2}$ and $T_{2}^{\prime}$.

We first construct $f_{1}$. Choose $S \in T_{1}$ and write $S=S_{0} 1^{b} k$ as above. Let $j$ be the last element of $S_{0}$. Define $S^{\prime}=f_{1}(S)=S_{0}(k-1)^{b}$. Let us check that $S^{\prime}$ belongs to $T_{1}^{\prime}$. It is easy to see that $S^{\prime}$ avoids 123. The length of $S^{\prime}$ is one less than the length of $S$, and the maximum of $S^{\prime}$ is equal to the maximum of $S$, hence $S^{\prime}$ has rank $n-1$. We know that $j \geq k$. In particular $j \neq k-1$, and hence $S_{0}$ is the longest prefix of $S^{\prime}$ whose last element is different from $k-1$. This shows that $S^{\prime} \in T_{1}^{\prime}$.

It is easy to check that $f_{1}$ can be inverted.

Let us now construct $f_{2}$. Choose $S \in T_{2}$, and write $S=S_{0} 1^{b} k$ as above. If $S_{0}$ is nonempty, let $j$ be the last element of $S_{0}$. Recall that no element of $S_{0}$ is smaller than $j$, and that $j$, if defined, is greater than 1 by definition of $S_{0}$. In particular, $S_{0}-1$ is a (possibly empty) sequence of positive numbers. Define $S^{\prime}=f_{2}(S)=\left(S_{0}-1\right)(k-1)^{b+1}$. The length of $S^{\prime}$ is equal to the length of $S$, and the maximum of $S^{\prime}$ is one less than the maximum of $S$, hence $S^{\prime}$ has rank $n-1$. It may be routinely checked that $S^{\prime}$ avoids 123 . Note that the last element of $S_{0}-1$ is smaller than $k-1$, and hence $S^{\prime}$ belongs to $T_{2}^{\prime}$. 
The inverse of $f_{2}$ is easy to obtain. Choose $S^{\prime} \in T_{2}^{\prime}$, with $S^{\prime}=S_{0}^{\prime}(k-1)^{b}$, where $S_{0}^{\prime}$ is the longest prefix of $S^{\prime}$ not ending with $k-1$. As we pointed out earlier, $S^{\prime}$ must end with the symbol $k-1$, hence $b \geq 1$. Define $S=\left(S_{0}+1\right) 1^{b-1} k$. It may be routinely checked that $S$ belongs to $T_{2}$.

The following results are direct consequences of Claim 57 and Claim 58. We omit their proofs.

Theorem 59. The number of 1123-avoiding matchings of size $n$ with last element equal to $k$ is equal to the number of Dyck paths of semilength $n$ whose last up-step is followed by $k$ down-steps.

Corollary 60. The number of 1123-avoiding matchings of size $n$ is $C_{n}=\frac{1}{n+1}\left(\begin{array}{c}2 n \\ n\end{array}\right)$. In particular, 1123 is equivalent to 1212 and to 1221.

From Theorem 59 we may derive the closed-form expression for $t(n, k)$. Since the number of Dyck paths that end with an up-step followed by $k$ down-steps is equal to the number of non-negative lattice paths from $(0,0)$ to $(2 n-k-1, k-1)$, we may apply standard arguments for the enumeration of non-negative lattice paths to obtain the formula

$$
t(n, k)=\frac{k}{n}\left(\begin{array}{c}
2 n-k-1 \\
n-1
\end{array}\right)
$$

\subsection{Classification of patterns of size 4}

Theorem 59 and the general results presented in the previous sections allow us to fully classify patterns of length four by their equivalence classes (see Table 2).

\begin{tabular}{l|l}
$\tau$ & $p(n ; \tau)$ \\
\hline \hline $1213,1223,1231,1232,1233,1234$ & {$[22$, Sequence A007051] (see Equation (2)) } \\
$1123,1212,1221$ & $\frac{1}{n+1}\left(\begin{array}{c}2 n \\
n\end{array}\right)[22$, Sequence A000108] (see Theorem 59) \\
1122 & $1,1,2,5,14,42,133,441, \ldots$ \\
$1112,1121,1211,1222$ & {$[22$, Sequence A005425] (see Corollary 37) } \\
1111 & {$[22$, Sequence A001680] (see Equation (1)) }
\end{tabular}

Table 2: Number of partitions in $P(n ; \tau)$, where $\tau \in P(4)$.

\section{The patterns of size five}

For a full characterization of the equivalence of patterns up to size seven, we need to consider one more isolated case, namely the pattern 12112. Our aim is to show that this pattern is equivalent to the three patterns 12221, 12212, and 12122. The latter three 
patterns are all equivalent by Corollary 40. It is thus sufficient to show that $12112 \sim$ 12212.

We remark that the proof involving the pattern 12112 does not use the notion of Ferrers equivalence. In fact, the matrix $M(2,112)$ is not Ferrers-equivalent to the three Ferrers-equivalent matrices $M(2,221), M(2,212)$ and $M(2,122)$.

\section{$5.1 \quad$ Introduction}

We will first introduce the basic terminology and notation that we will use throughout the proof.

Let $S=s_{1} s_{2} \cdots s_{n}$ be a sequence of length $n$ over the alphabet $[m]$, such that every element of $[\mathrm{m}]$ appears in $S$ at least once. For $i \in[\mathrm{m}]$ let $f_{i}$ and $\ell_{i}$ denote the index of the first and the last symbol of $S$ that is equal to $i$. Formally, $f_{i}=\min \left\{j: s_{j}=i\right\}$ and $\ell_{i}=\max \left\{j: s_{j}=i\right\}$.

Definition 61. For $k \in[\mathrm{m}]$, we say that the sequence $S$ is a $k$-semicanonical sequence ( $k$-sequence for short), if $S$ has the following properties.

- For every $i$ and $j$ such that $1 \leq i<k$ and $i<j$, we have $f_{i}<f_{j}$.

- For every $i$ and $j$ such that $k \leq i<j \leq m$, we have $\ell_{i}<\ell_{j}$.

Note that $m$-semicanonical sequences are precisely the canonical sequences of partitions of $[n]$ with $m$ blocks (i.e., the sequences satisfying $f_{i}<f_{i+1}$ for $i \in[m-1]$ ), while the 1 -canonical sequences are precisely the sequences satisfying $\ell_{i}<\ell_{i+1}$ for $i \in[m-1]$. Note that for every fixed $k \in[m]$ and a fixed partition $\pi=\pi_{1} \cdots \pi_{n}$ with $m$ blocks, there is exactly one $k$-sequence $S=s_{1} \cdots s_{n}$ with the property $s_{i}=s_{j} \Longleftrightarrow \pi_{i}=\pi_{j}$.

In particular, assuming $n$ and $m$ are fixed, the number of $k$-sequences is independent of $k$, and each partition of $[n]$ with $m$ blocks is represented by a unique $k$-sequence. To prove the equivalence $12112 \sim 12212$, we will exploit a remarkable property of the pattern 12112 , described by the following key lemma.

Lemma 62. For every fixed $n$ and $m$, the number of 12112-avoiding $k$-sequences is independent of $k$. Thus, for every $k \in[m]$, the number of 12112-avoiding $k$-sequences of length $n$ with $m$ symbols is equal to the number of 12112-avoiding partitions of $n$ with $m$ blocks.

Before we prove of Lemma 62, let us explain how the lemma implies the equivalence $12112 \sim 12212$.

Theorem 63. The pattern 12112 is equivalent to 12212. In fact, for every $m$ and $n$, there is a bijection between 12112-avoiding partitions of $[n]$ with $m$ blocks and 12212-avoiding partitions of $[n]$ with $m$ blocks.

Proof. Fix $m$ and $n$. We know that the 12112-avoiding partitions of $[n]$ with $m$ blocks correspond precisely to $m$-semicanonical sequences over $[m]$ of length $n$, and by Lemma 62 , 
these sequences are in bijection with 1-semicanonical 12112-avoiding sequences of the same length and alphabet. It remains to provide a bijection between the 12112-avoiding 1-sequences and the 12212-avoiding partitions.

Take a 1-semicanonical 12112-avoiding sequence $S$ with $m$ symbols and length $n$, reverse the order of letters in $S$, and then replace each symbol $i$ of the reverted sequence by the symbol $m-i+1$. It is easy to check that this transform is an involution which maps 12112-avoiding 1-sequences onto 12212-avoiding $m$-sequences, which are precisely the 12212-avoiding partitions of $[n]$ with $m$ blocks.

It now remains to prove Lemma 62. For the rest of the proof, unless otherwise noted, we will assume that $m$ and $n$ are fixed, and that each sequence we consider has length $n$ and $m$ distinct symbols.

In the following arguments, it is often convenient to represent a sequence $S=s_{1} \cdots s_{n}$ by the matrix $M(S, m)$. Recall that $M(S, m)$ is the $0-1$ matrix with $m$ rows and $n$ columns, with a 1-cell in row $i$ and column $j$ if and only if $s_{j}=i$. A matrix representing a $k$-sequence will be called $k$-semicanonical matrix (or just $k$-matrix), and a matrix representing a 12112-avoiding sequence will be simply called 12112-avoiding matrix. In accordance with earlier terminology, we will use the term sparse matrix for a 0-1 matrix with at most one 1-cell in each column, and we will use the term semi-standard matrix for a 0-1 matrix with exactly one 1-cell in each column. For a 0-1 matrix $M$, we let $f_{i}(M)$ and $\ell_{i}(M)$ denote the column-index of the first and the last 1-cell in the $i$-th row of $M$. We will write $f_{i}$ and $\ell_{i}$ instead of $f_{i}(M)$ and $\ell_{i}(M)$ if there is no risk of confusion.

Before we formulate the proof of Lemma 62, let us present a brief sketch of the main idea. We will first build a bijection that transforms a $(k+1)$-matrix $M$ into a $k$-matrix, ignoring 12112-avoidance for a while. Let the last 1-cell in row $k$ of $M$ be in column $c$, let us call the row $k$ the key row of $M$. If the last 1-cell in row $k+1$ appears to the right of column $c$, then $M$ is already a $k$-matrix and we are done. On the other hand, if row $k+1$ has no 1-cell to the right of $c$, we swap the key row $k$ with the row $k+1$, to obtain a new matrix $M^{\prime}$ whose key row is now the row $k+1$. We repeat this procedure until we reach the situation when the key row is either the topmost row of the matrix, or the row above the key row has a 1-cell to the right of column $c$. This procedure transforms the original $k+1$ matrix into a $k$-matrix. Also, the procedure is invertible (note that the first 1-cell of the key row is always to the left of any other 1-cell in the rows $k, k+1, \ldots, m$ ).

Unfortunately, this simplistic approach does not preserve 12112-avoidance. However, we will present an algorithm which follows the same basic structure as the procedure above, but instead of merely swapping the key row with the row above it, it performs a more complicated step. The description of this step is the main ingredient of our proof.

To formalize our argument, we need to introduce more definitions. Let $M$ be a $0-1$ matrix with exactly one 1-cell in each column and at least one 1-cell in each row, and let us write $f_{i}=f_{i}(M)$ and $\ell_{i}=\ell_{i}(M)$. Let $k, p$ and $q$ be three row-indices of $M$, with $k \leq p \leq q$. We will say that $M$ is a $(k, p, q)$-matrix, if $M$ satisfies the following conditions.

- The matrix obtained from $M$ by erasing row $p$ is a $k$-semicanonical matrix with $m-1$ rows. 
- For each $i<k$, we have $f_{i}<f_{p}$. For every $j \geq k, j \neq p$, we have $f_{p}<f_{j}$.

- The number $q$ is determined by the relation $q=\max \left\{j: \ell_{j} \leq \ell_{p}\right\}$. Thus, the first condition implies that $\ell_{j} \leq \ell_{p}$ for every $j \in\{k, k+1, \ldots, q\}$.

In a $(k, p, q)$-matrix, row $p$ will be called the key row.

Intuitively, a $(k, p, q)$-matrix is an intermediate stage of the above-described procedure which transforms a $(k+1)$-matrix into a $k$-matrix by moving the key row towards the top. The number $p$ is the index of the key row in a given step of the procedure, while the number $q$ is the topmost row that needs to be swapped with the key row to produce the required $k$-matrix. In particular, a matrix $M$ is $(k+1)$-semicanonical if and only if it is a $(k, k, q)$-matrix for some value of $q$, and $M$ is $k$-semicanonical if and only if it is a $(k, q, q)$-matrix for some $q$.

As an example, consider the sequence $S=1331232431$ with $n=10$ and $m=4$. This sequence corresponds to the following matrix $M=M(S, 4)$.

$$
M=\left(\begin{array}{l}
0000000100 \\
0110010010 \\
0000101000 \\
1001000001
\end{array}\right) \quad M^{\prime}=\left(\begin{array}{l}
0110010010 \\
0000000100 \\
0000101000 \\
1001000001
\end{array}\right)
$$

The matrix $M$ is a $(2,3,4)$-matrix. If we exchange the third row (which acts as the key row) with the fourth row, we obtain a $(2,4,4)$-matrix $M^{\prime}$ representing the 2 -sequence $S^{\prime}=1441242341$. The matrix $M^{\prime}$ can also be regarded as a $(1,1,4)$-matrix, with the key row at the bottom.

The following lemma implies Lemma 62.

Lemma 64. For arbitrary $k \leq p<q$, there is a bijection $\phi$ between 12112-avoiding $(k, p, q)$-matrices and 12112-avoiding $(k, p+1, q)$-matrices.

Before we construct the bijection $\phi$, we need to prove several basic properties of the 12112-avoiding $(k, p, q)$-matrices.

\subsection{Tools of the proof}

Let us introduce some more terminology. If $x \in[m]$ is a row of a matrix $M$, then an $x$-column is a column of $M$ that has a 1-cell in row $x$. Similarly, if $X \subseteq[m]$ is a set of rows of $M$, we will say that a column $j$ is an $X$-column if $j$ has a 1-cell in a row belonging to $X$.

If $x, y$ is a pair of rows of $M$ with $x<y$, we will say that $M$ contains 12112 in $(x, y)$ if the submatrix of $M$ induced by the pair of rows $x, y$ contains 12112. If $X$ and $Y$ are two sets of rows, we will say that $M$ contains 12112 in $(X, Y)$ if there is an $x \in X$ and $y \in Y$ such that $x<y$ and $M$ contains 12112 in $(x, y)$.

Throughout this section, we will assume that $k, p, q$ are fixed, and that $k \leq p<q$.

We now state a pair of simple but useful observations. Their proofs are straightforward, and we omit them. 
Observation 65. Let $M$ be a sparse 0-1 matrix, and let $x<y$ be two rows of $M$, such that $f_{x}<f_{y}$. The matrix $M$ avoids 12112 in $(x, y)$ if and only if $M$ has at most one $x$-column s satisfying $f_{y}<s<\ell_{y}$. If such a unique column $s$ exists, we will say that $s$ separates row $y$. The $y$-columns that are to the left of the separating column $s$ will be called front $y$-columns (with respect to row $x$ ) and their 1-cells will be called front 1-cells. Similarly, the y-columns to the right of $s$ will be called rear $y$-columns and their 1-cells are rear 1-cells. If there is no such separating column, then we will assume that all the $y$-columns and their 1-cells are front.

Observation 66. Let $M$ be a sparse 0-1 matrix, and let $x<y$ be a pair of rows such that $\ell_{x}<\ell_{y}$. Let $t$ be the number of 1-cells in row $x$, and let $c_{i}$ be the $i$-th $x$-column, i.e., $f_{x}=c_{1}<c_{2}<\cdots<c_{t}=\ell_{x}$. The matrix $M$ avoids 12112 in $(x, y)$, if and only if every $y$-column appears either to the left of column $c_{1}$, or between the columns $c_{t-1}$ and $c_{t}$, or to the right of column $c_{t}$. These three types of y-columns (and their 1-cells) will be called left, middle, and right $y$-columns (or 1-cells) with respect to row $x$.

Lemma 67. Let $M$ be a 12112-avoiding $(k, p, q)$-matrix, and let $j$ be a row of $M$ with $k \leq j \leq p$. Let $M^{\prime}$ be a sparse 0-1 matrix of the same size as $M$, with the property that for every $i \notin\{j, j+1, \ldots, q\}$, the $i$-th row of $M$ is equal to the $i$-th row of $M^{\prime}$. If $M^{\prime}$ has a copy of the pattern 12112 in a pair of rows $x<y$, then $j \leq x \leq q$.

Proof. Let $M$ and $M^{\prime}$ be as above. We will call the rows $\{j, j+1, \ldots, q\}$ mutable, and the remaining rows will be called constant.

Assume that $M^{\prime}$ has a copy of 12112 in the rows $x<y$. Clearly, at least one of the two rows $x, y$ must be mutable, and in particular, we must have $x \leq q$. The lemma claims that $x$ must be mutable. For contradiction, assume that $x<j$. There are two possibilities; either $x<k$ or $k \leq x<j$.

If $x<k$, then $y$ is one of the mutable rows. From the definition of the $(k, p, q)$-matrix, we obtain that all the columns of $M$ to the left of $f_{p}(M)$ and to the right of $\ell_{p}(M)$ contain a 1-cell in one of the constant rows. Since $M^{\prime}$ is sparse, we conclude that in $M^{\prime}$, all the 1-cells in the mutable rows can only appear in the columns $i$ such that $f_{p}(M) \leq i \leq \ell_{p}(M)$.

Now, we apply Observation 65 to the rows $x$ and $p$ in the matrix $M$, and conclude that $M$ (and hence also $M^{\prime}$ ) has at most one $x$-column $s$ such that $f_{p}(M) \leq s \leq \ell_{p}(M)$. Therefore $M^{\prime}$ also has at most one $x$-column between $f_{y}\left(M^{\prime}\right)$ and $\ell_{y}\left(M^{\prime}\right)$. By Observation 65, this shows that $x$ cannot form the pattern 12112 with any of the mutable rows $y$ of $M^{\prime}$.

Assume now that $k \leq x<j$. As before, we have $y \in\{j, \ldots, q\}$. Let $c_{1}<c_{2}<\cdots<c_{t}$ be the $x$-columns of $M$ (and hence of $M^{\prime}$ as well, since $x$ is constant). For any mutable row $i$, we have $\ell_{x}(M)<\ell_{i}(M)$ by the definition of $(k, p, q)$-matrix. By Observation 66, all of the $i$-columns of $M$ appear either to the left of $c_{1}$ or to the right of $c_{t-1}$. In particular, all the 1-cells between the columns $c_{1}$ and $c_{t-1}$ belong to the constant rows. This implies that $M^{\prime}$ can have no occurrence of 12112 in the two rows $x<y$.

We will now describe a simple operation, call pseudoswap, on 12112-avoiding pairs of rows. 


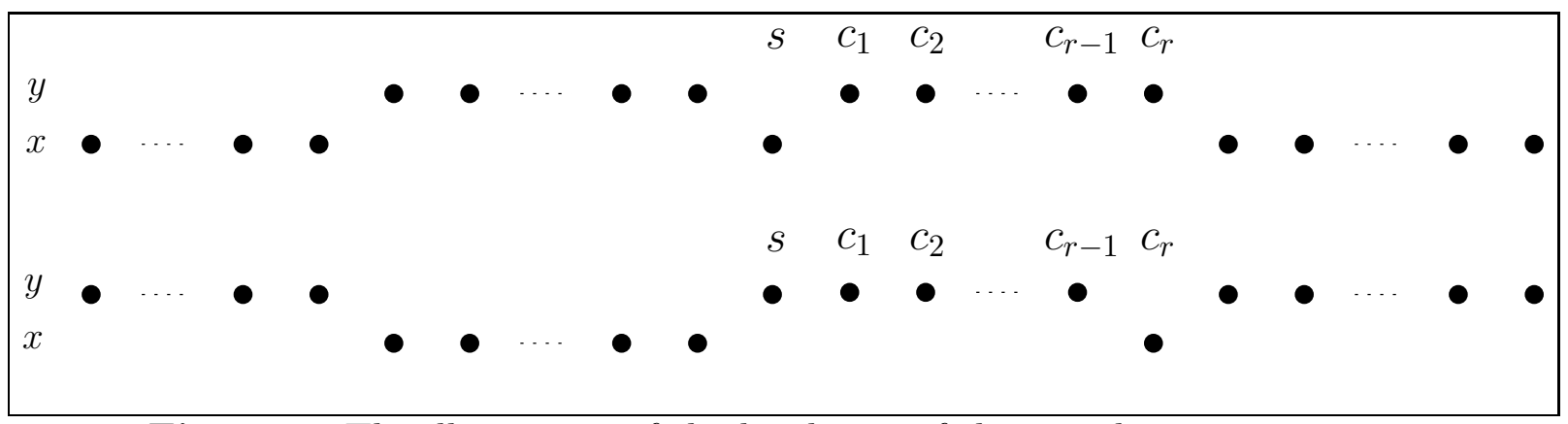

Figure 2: The illustration of the hard case of the pseudoswap operation.

Assume that $M$ is a sparse matrix with a pair of adjacent rows $x$ and $y=x+1$ that avoids 12112 in $(x, y)$. Assume furthermore that $f_{x}<f_{y} \leq \ell_{y}<\ell_{x}$. The pseudoswap of the two rows is performed as follows.

Easy case. If the row $y$ is not separated by an $x$-column (in the sense of Observation 65), or if $M$ has at most one rear $y$-column with respect to row $x$, the pseudoswap is performed by simply swapping the two rows.

Hard case. Assume $M$ has an $x$-column $s$ separating $y$, and that it has $r>1$ rear $y$ columns $c_{1}<c_{2}<\cdots<c_{r}$ (see Figure 2). In this case, the pseudoswap preserves the position of all the 1-cells in columns $c_{1}, \ldots, c_{r-1}$ (i.e., the 1-cells in these columns remain in row $y$ ), and all the other 1-cells in rows $x, y$ are moved from $x$ to $y$ and vice versa. Note that after the pseudoswap is performed, the columns $s<c_{1}<c_{2}<$ $\cdots<c_{r-1}$ all contain a 1-cell in row $y$, and these $r$ 1-cells are precisely the middle 1 -cells of $y$ with respect to $x$. (in the sense of Observation 66).

Let $M^{\prime}$ be the matrix obtained from $M$ by the pseudoswap. It can be routinely checked that $M^{\prime}$ avoids 12112 in $(x, y)$. Let us write $f_{i}^{\prime}$ for $f_{i}\left(M^{\prime}\right)$ and $\ell_{i}^{\prime}$ for $\ell_{i}\left(M^{\prime}\right)$. Clearly, $f_{x}^{\prime}=f_{y}$ and $f_{y}^{\prime}=f_{x}$, and also $\ell_{x}^{\prime}=\ell_{y}$ and $\ell_{y}^{\prime}=\ell_{x}$. Also, if $M$ has $r \geq 0$ rear cells in row $y$, then $M^{\prime}$ has $r$ middle cells in row $y$.

It is not difficult to see that the pseudoswap can be inverted. Let $M^{\prime}$ be a sparse matrix avoiding 12112 in two adjacent rows $x<y$, such that $f_{y}^{\prime}<f_{x}^{\prime} \leq \ell_{x}^{\prime}<\ell_{y}^{\prime}$. If $M^{\prime}$ has fewer than two middle $y$-columns, we invert the easy case of the pseudoswap by exchanging the two rows. On the other hand, if $M^{\prime}$ has $r>1$ middle $y$-columns $m_{1}<\cdots<m_{r}$, we invert the hard case by preserving the position of the 1-cells in columns $m_{2}, m_{3}, \ldots, m_{r}$ and inverting all the other $\{x, y\}$-columns.

We will be mostly interested in the situation when the pseudoswap is applied to the pair of rows $(p, p+1)$ in a $(k, p, q)$-matrix with $p<q$. It is not hard to see that this operation yields a $(k, p+1, q)$-matrix. Let us now look in more detail at the situation related to the hard case of the pseudoswap. Recall that if $X$ and $Y$ are two sets of rows of $M$, we say that $M$ avoids 12112 in $(X, Y)$, if there is no $x \in X$ and $y \in Y$ such that $x<y$ and the two rows $x, y$ contain a copy of 12112 .

The following lemma is illustrated in Figure 3. 
Lemma 68. (a) Let $M$ be a $(k, p, q)$-matrix that avoids 12112 in $(p, p+1)$. Let $f_{p}(M)=$ $b_{1}<b_{2}<\cdots<b_{t}=\ell_{p}(M)$ be the $p$-columns of $M$. Assume that the row $p+1$ is separated by the column $b_{i}$, and that it has $r \geq 2$ rear 1 -cells. Let $c_{1}<c_{2}<\cdots<c_{s}$ be the front $(p+1)$-columns and let $d_{1}<d_{2}<\cdots<d_{r}$ be the rear $(p+1)$-columns. By Observation 65, we have the inequalities

$$
b_{1}<\cdots<b_{i-1}<c_{1}<\cdots<c_{s}<b_{i}<d_{1}<\cdots<d_{r}<b_{i+1}<\cdots<b_{t} .
$$

Let $X=\{p, p+1\}$ and let $Y$ be the set of all the rows above $p+1$ that contain at least one 1-cell to the left of the column $d_{r-1}$; formally,

$$
Y=\left\{y>p+1: \quad f_{y}(M)<d_{r-1}\right\}
$$

The matrix $M$ avoids 12112 in $(X, Y)$ if and only if each $Y$-column $y$ satisfies one of the following three inequalities:

1. $b_{i-1}<y<c_{1}=f_{p+1}$

2. $d_{r-1}<y<d_{r}$

3. $d_{r}<y<b_{i+1}$

The rows in $Y$ are precisely the rows above $p+1$ that are separated by the $p$-column $b_{i}$.

(b) Let $M^{\prime}$ be a $(k, p+1, q)$-matrix that avoids 12112 in $(p, p+1)$. Let $\alpha_{1}<\cdots<$ $\alpha_{u}<\beta_{1}<\cdots<\beta_{r}<\gamma_{1}<\cdots<\gamma_{v}$ be the $(p+1)$-columns of $M^{\prime}$, where the $\alpha_{i}$, $\beta_{i}$ and $\gamma_{i}$ denote respectively the left, middle and right $(p+1)$-columns with respect to row $p$. Assume that there are at least two middle 1-cells. Let $\delta_{1}<\cdots<\delta_{w}$ be the p-columns of $M^{\prime}$. By Observation 66, we have the inequalities

$$
\alpha_{1}<\cdots<\alpha_{u}<\delta_{1}<\cdots<\delta_{w-1}<\beta_{1}<\cdots<\beta_{r}<\delta_{w}<\gamma_{1}<\cdots<\gamma_{v} .
$$

Let $X=\{p, p+1\}$ and let $Y^{\prime}$ be the set of all the rows above $p+1$ that contain at least one 1-cell to the left of column $\beta_{r}$. The matrix $M^{\prime}$ avoids 12112 in $\left(X, Y^{\prime}\right)$ if and only if each $Y^{\prime}$-column y satisfies one of the following three inequalities:

1. $\beta_{r-1}<y<\beta_{r}$

2. $\beta_{r}<y<\delta_{w}$

3. $\delta_{w}<y<\gamma_{1}$

The rows in $Y^{\prime}$ are precisely the rows above $p+1$ that are separated by the $(p+1)$ column $\beta_{r}$. 


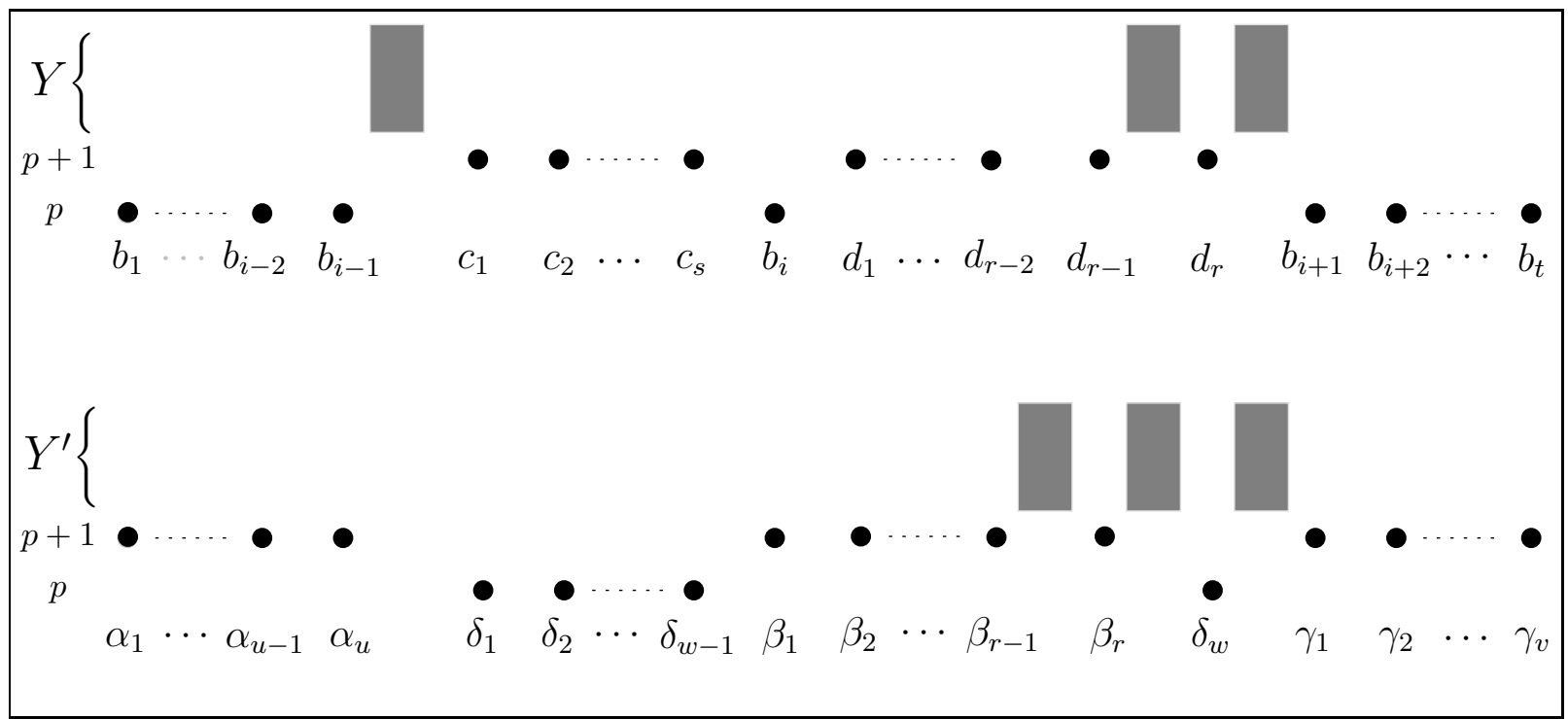

Figure 3: Illustration of Lemma 68, part $(a)$ is above, part $(b)$ below. The black dots correspond to 1-cells in rows $p$ and $p+1$, and the grey rectangles correspond to possible positions of the 1-cells in the rows of $Y$ or $Y^{\prime}$.

Proof. Let us consider part $(a)$. Fix a row $y \in Y$. By the definition of a $(k, p, q)$-matrix, we have $d_{r}<\ell_{y}$. By Observation 66, we see that $M$ avoids 12112 in $(p+1, y)$ if and only if every $y$-column $j$ satisfies either $j<c_{1}=f_{p+1}, d_{r-1}<j<d_{r}$, or $j>d_{r}$. The first $y$-column satisfies $f_{y}<d_{r-1}$ by the definition of $Y$, and hence $f_{y}<c_{1}=f_{p+1}<b_{i}$. Since $\ell_{y}>\ell_{p+1}=d_{r}>b_{i}$, we see that if the pair of rows $(p+1, y)$ avoids 12112 , then $y$ is separated by $b_{i}$ and the two rows $(p, y)$ avoid 12112 if and only if $b_{i-1}<f_{y}<\ell_{y}<b_{i+1}$. This proves part $(a)$ of the lemma.

The proof of part $(b)$ is analogous and we omit it.

\subsection{The bijection}

We are now ready to present the bijection $\phi$. Let $M$ be a 12112 -avoiding $(k, p, q)$-matrix with $p<q$, and let us write $f_{i}$ and $\ell_{i}$ for $f_{i}(M)$ and $\ell_{i}(M)$. By the definition of $(k, p, q)$ matrix and by the assumption $p<q$, we know that $f_{p}<f_{p+1} \leq \ell_{p+1}<\ell_{p}$, so we may perform the pseudoswap of the rows $p$ and $p+1$ in $M$. Let $M^{\prime}$ be the $m \times n$ matrix obtained from $M$ by this pseudoswap. Let $f_{i}^{\prime}=f_{i}\left(M^{\prime}\right)$ and $\ell_{i}^{\prime}=\ell_{i}\left(M^{\prime}\right)$. Note that $f_{i}^{\prime}=f_{i}$ and $\ell_{i}^{\prime}=\ell_{i}$ for every $i \notin\{p, p+1\}$.

Clearly, $M^{\prime}$ is a $(k, p+1, q)$-matrix. We now distinguish two cases, depending on whether the pseudoswap we performed was easy or hard.

Easy case. If the row $p+1$ of $M$ has at most one rear 1 -cell with respect to row $p$, then $M^{\prime}$ is 12112-avoiding, and we may define $\phi(M)=M^{\prime}$. Indeed, from the definition of the pseudoswap we know that $M^{\prime}$ cannot contain a copy of 12112 in the rows $(p, p+1)$, and since we are performing the easy case of the pseudoswap, we cannot create any new copy of the forbidden pattern that would intersect the remaining $m-2$ rows. 
Hard case. Assume that the row $p+1$ of $M$ has $r>1$ rear 1 -cells. Let $b_{1}<\cdots<b_{t}$, $c_{1}<\cdots<c_{s}, d_{1}<d_{2}<\cdots<d_{r}$, and $Y$ have the same meaning as in part $(a)$ of Lemma 68. Let $Y_{1}, Y_{2}$ and $Y_{3}$ denote, respectively, the $Y$-columns that lie between $b_{i-1}$ and $c_{1}$, between $d_{r-1}$ and $d_{r}$, and between $d_{r}$ and $b_{i+1}$.

The bijection $\phi$ is now constructed in two steps. In the first step, we perform the pseudoswap of the rows $p$ and $p+1$. Let $M^{\prime}$ be the result of this first step. Let us now apply the notation of part $(b)$ of Lemma 68 to the matrix $M^{\prime}$ (see Figure 3). Note that $d_{r-1}=\beta_{r}$, and hence $Y=Y^{\prime}$. Part $(b)$ of Lemma 68 requires that all the $Y^{\prime}$-columns of a 12112-avoiding $(k, p+1, q)$-matrix fall into one of the three groups:

- columns between $\delta_{w}<y<\gamma_{1}$. In $M^{\prime}$, we have $\delta_{w}=d_{r}$ and $\gamma_{1}=b_{i+1}$, so these columns are precisely the columns in $Y_{3}$.

- columns between $\beta_{r}<y<\delta_{w}$. In $M^{\prime}$, these are precisely the columns in $Y_{2}$.

- columns between $\beta_{r-1}<y<\beta_{r}$. In $M^{\prime}$, there are no $Y$-columns in this range.

On the other hand, if $Y_{1}$ is nonempty, then these columns violate the inequalities of part (b) in Lemma 68, showing that $M^{\prime}$ is not 12112-avoiding. To correct this, we apply the second step of the bijection $\phi$. Consider the submatrix of $M^{\prime}$ induced by the columns $Y_{1}$ and the columns $Z=\left\{\delta_{1}<\cdots<\delta_{w-1}<\beta_{1}<\cdots<\beta_{r-1}\right\}$. Note that the columns $Y_{1}$ are to the left of any column of $Z$. Now we rearrange the columns inside this submatrix, so that all the columns in $Y_{1}$ appear after the columns in $Z$, keeping the relative order of the columns in $Y_{1}$, as well as those in $Z$. This transforms $M^{\prime}$ into a matrix $M^{\prime \prime}$. We define $\phi(M)=M^{\prime \prime}$.

Since $M^{\prime \prime}$ is clearly a $(k, p+1, q)$-matrix, it remains to check that $M^{\prime \prime}$ avoids 12112 . Let $x<y$ be a pair of rows of $M^{\prime \prime}$. We want to check that $M^{\prime \prime}$ avoids 12112 in these two rows. Let us consider the following cases separately.

The case $x<p$. The rows below row $p$ are unaffected by $\phi$. The rows above row $q$ are preserved as well, because any row $z \in Y$ must satisfy $\ell_{z}<b_{i+1} \leq \ell_{p}$, so no row above $q$ belongs to $Y$. Thus, we may apply Lemma 67, to see that $M^{\prime \prime}$ avoids 12112 in the rows $(x, y)$.

The case $x=p, y=p+1$. The properties of pseudoswap guarantee that $M^{\prime \prime}$ avoids 12112 in these two rows.

The case $x \in X=\{p, p+1\}$ and $y \in Y^{\prime}$. By construction, $M^{\prime \prime}$ satisfies the inequalities of part $(b)$ of Lemma 68 , and thus it avoids 12112 in $(X, Y)$.

The case $x \in X=\{p, p+1\}, y \notin Y^{\prime}$ and $y>p+1$. By the definition of $Y^{\prime}$, we have $f_{y}\left(M^{\prime \prime}\right)=f_{y}(M)>d_{r-1}=\beta_{r}$. In any column to the right of $\beta_{r}$ the mapping $\phi$ acts by exchanging the rows $p$ and $p+1$. It is easy to check that this action cannot create a copy of 12112 in $(x, y)$ (note that in any of the three matrices $M, M^{\prime}$ and $M^{\prime \prime}$, both the rows $p$ and $p+1$ have a 1 -cell to the left of $\beta_{r}$ ).

The case $y>x>p+1$. The submatrix of $M^{\prime \prime}$ induced by the rows above $p+1$ only differs from the corresponding submatrix of $M$ by the position of the zero columns. Thus, it cannot contain any copy of 12112 .

This shows that $\phi(M)$ is indeed a 12112 -avoiding $(k, p+1, q)$-matrix. 
It is routine to check that the mapping $\phi$ can be inverted, which shows that $\phi$ is indeed the required bijection.

The following corollary is a direct consequence of Theorem 63 and Lemma 10.

Corollary 69. For every $r$ and $c$, there is a bijection between the $M(2,112)$-avoiding semistandard fillings of all the Ferrers shapes with $r$ rows and c columns and the $M(2,212)$ avoiding semi-standard fillings of all the Ferrers shapes with $r$ rows and c columns.

It would be tempting to assume that the two matrices $M(2,112)$ and $M(2,212)$ are Ferrers-equivalent. However, as we already mentioned in the introduction of Section 5, this is not the case. For instance, the Ferrers shape $F$ with five columns of height 4 and one column of height 2 has $866 M(2,112)$-avoiding fillings but only $865 M(2,212)$-avoiding fillings. Thus, the bijection of Corollary 69 in general cannot preserve the shape of the underlying diagram.

\subsection{Classification of patterns of size 5}

In Table 3, we list the equivalence classes of patterns of size 5, together with the reference to the appropriate result. By Corollary 32, if $\tau$ and $\sigma$ are two equivalent patterns of size 4 , then $1(\tau+1)$ and $1(\sigma+1)$ are equivalent patterns of size 5 , and vice versa. Thus, the classification of the patterns of size 4 , given in Table 2, explains the equivalences among patterns of size 5 with only one occurrence of 1 . For this reason, Table 3 only lists the references for the patterns with at least two occurrences of the symbol 1.

Table 3: Classification of patterns of size five.

\begin{tabular}{|l|l|l|}
\hline$\tau$ & $p(11 ; \tau)$ & Reference \\
\hline \hline $\begin{array}{l}12314,12324,12334,12341, \\
12342,12343,12344,12345\end{array}$ & 175275 & Theorem 33 \\
\hline 12313 & 213423 & \\
\hline $\begin{array}{l}12123,12132,12134,12213, \\
12231,12234,12312,12321, \\
12323,12331,12332\end{array}$ & 223191 & $\begin{array}{l}12332 \sim 12331 \sim 12321 \text { by Proposition } 15,12234 \sim 12134 \\
\text { by Corollary } 13, \text { the rest by Theorem } 48\end{array}$ \\
\hline 12133,12233 & & \\
\hline 11223,11232 & 238379 & Corollary 13 \\
\hline 11234 & 276670 & Theorem 41 \\
\hline 12131 & 282503 & \\
\hline 11233 & 285503 & \\
\hline $12113,12223,12232,12311$, & 288543 & $12311 \sim 12322$ and $12113 \sim 12223$ by Corollary 13 \\
12322,12333 & 288157 & \\
\hline 11231 & 322218 & \\
\hline 11213 & 323663 & \\
\hline 11123 & 348887 & \\
\hline $12112,12122,12212,12221$ & 362447 & $12112 \sim 12212$ by Theorem 63, the rest by Corollary 40 \\
\hline 12121 & 364317 & \\
\hline 11212 & 364341 & \\
\hline 12211 & 373270 & \\
\hline 11221 & 376556 & \\
\hline
\end{tabular}




\begin{tabular}{|l|l|l|}
\hline 11222 & 378365 & \\
\hline 11122 & 379805 & \\
\hline $\begin{array}{l}11112,11121,11211,12111, \\
12222\end{array}$ & 441009 & Theorems 34 and 36 \\
\hline 11111 & 556711 & \\
\hline
\end{tabular}

\section{The patterns of size six}

In Table 4, we list the equivalence classes of patterns of size 6 . To save space, we omit the singleton equivalence classes and the enumeration data. The full enumeration of these patterns is available from the second author's website [27]. As before, we provide references for the patterns that contain at least two occurrences of the symbol 1.

Table 4: Nonsingleton equivalence classes of patterns of size six.

\begin{tabular}{|l|l|}
\hline Pattern & Reference \\
\hline \hline $123415,123425,123435,123445$, & Theorem 33 \\
$123451,123452,123453,123454$, & \\
123455,123456 & \\
\hline 123413,123424 & Corollary 13 \\
\hline 123134,123143 & Theorem 54 \\
\hline $123124,123145,123214,123234$, & $123421 \sim 123431 \sim 123441 \sim 123432$ by Proposition $15,123124 \sim$ \\
$123243,123245,123324,123341$, & $123234,123145 \sim 123245,123214 \sim 123324,123341 \sim 123342$ and \\
$123342,123345,123412,123421$, & $123412 \sim 123423$ by Corollary 13 \\
$123423,123431,123432,123434$, & \\
$123441,123442,123443$ & \\
\hline $123144,123244,123344$ & Corollary 13 \\
\hline $121334,122334,121343,122343$ & $121334 \sim 122334$ and $121343 \sim 122343$ by Corollary 13 \\
\hline 121345,122345 & Corollary 13 \\
\hline 121344,122344 & Corollary 13 \\
\hline $123114,123224,123334,123343$, & $123114 \sim 123224$ and $123411 \sim 123422$ by Corollary 13 \\
$123411,123422,123433,123444$ & \\
\hline 121234,122134 & Corollary 40 \\
\hline $123123,123312,123321$ & Corollary 20 \\
\hline 122133,121233 & Corollary 40 \\
\hline 112334,112343 & Theorem 41 \\
\hline 121134,122234 & Corollary 13 \\
\hline $121223,121232,121322,122123$, & $123112 \sim 123223$ by Corollary $13,123331 \sim 123332 \sim 123221$ by \\
\hline $122132,122213,122231,122312$, & Proposition 15, the rest by Theorem 48 \\
\hline $122321,123112,123122,123212$, & \\
\hline $123221,123223,123233,123323$, & \\
123331,123332 & \\
\hline 123121,123232 & Corollary 13 \\
\hline $122311,123311,123211,123322$ & $122311 \sim 123211$ by Theorem 42, the rest by Proposition 15 \\
\hline 121333,122333 & Corollary 13 \\
\hline 121133,122233 & Corollary 13 \\
\hline $112223,112232,112322$ & Theorem 41 \\
\hline
\end{tabular}


Table 4: Nonsingleton equivalence classes of patterns of size six.

\begin{tabular}{|l|l|}
\hline Pattern & Reference \\
\hline \hline $121113,122232,122322,123111$, & $121113 \sim 122223$ and $123111 \sim 123222$ by Corollary 13 \\
$123222,123333,122223$ & \\
\hline 111223,111232 & Theorem 41 \\
\hline $122221,121222,122122,122212$ & Corollary 40 \\
\hline $111112,111121,111211,112111$, & Theorems 34 and 36 \\
121111,122222 & \\
\hline
\end{tabular}

\section{The patterns of size seven}

Table 5 lists the nonsingleton classes of patterns of size 7 . The full listing of all the classes, together with enumeration data that shows the distinction between the classes, is available from the second author's website [28].

Table 5: Nonsingleton equivalence classes of patterns of size seven.

\begin{tabular}{|c|c|}
\hline $\begin{array}{l}\text { Pattern } \\
\end{array}$ & Reference \\
\hline $\begin{array}{l}1234516,1234526,1234536,1234546, \\
1234556,1234561,1234562,1234563, \\
1234564,1234565,1234566,1234567\end{array}$ & Theorem 33 \\
\hline 1234514,1234525 & Corollary 13 \\
\hline $1234513,1234524,1234535$ & Corollary 13 \\
\hline $1234135,1234245,1234254$ & $1234135 \sim 1234245$ by Corollary 13 \\
\hline 1234351,1234352 & Corollary 13 \\
\hline 1234315,1234425 & Corollary 13 \\
\hline $\begin{array}{l}\text { 1234125, 1234156, 1234215, 1234235, } \\
\text { 1234256, 1234325, 1234345, 1234354, } \\
\text { 1234356, 1234435, 1234451, 1234452, } \\
\text { 1234453, 1234456, 1234512, 1234521, } \\
1234523,1234531,1234532,1234534, \\
1234541,1234542,1234543,1234545, \\
1234551,1234552,1234553,1234554\end{array}$ & $\begin{array}{l}1234521 \sim 1234531 \sim 1234541 \sim 1234551 \sim 1234532 \text { by } \\
\text { Proposition } 15,1234125 \sim 1234235,1234156 \sim 1234256, \\
1234215 \sim 1234325,1234451 \sim 1234452 \text { and } 1234512 \sim \\
1234523 \text { by Corollary } 13\end{array}$ \\
\hline $1234155,1234255,1234355,1234455$ & Corollary 13 \\
\hline 1233451,1233452 & Corollary 13 \\
\hline $\begin{array}{l}\text { 1231445, 1231454, 1232445, 1232454, } \\
1233445,1233454\end{array}$ & Corollary 13 \\
\hline $1231456,1232456,1233456$ & Corollary 13 \\
\hline $1231455,1232455,1233455$ & Corollary 13 \\
\hline $\begin{array}{l}1234115,1234225,1234335,1234445, \\
1234454,1234511,1234522,1234533, \\
1234544,1234555\end{array}$ & Corollary 13 \\
\hline $1231245,1232145,1232345,1233245$ & $\begin{array}{l}1231245 \sim 1232345 \text { by Corollary } 13,1231245 \sim 1232145 \text { by } \\
\text { Corollary } 40\end{array}$ \\
\hline 1234132,1234243 & Corollary 13 \\
\hline 1234213,1234324 & Corollary 13 \\
\hline
\end{tabular}


Table 5: Nonsingleton equivalence classes of patterns of size seven.

\begin{tabular}{|c|c|}
\hline Pattern & Reference \\
\hline $\begin{array}{l}1231234,1233214,1233412,1233421, \\
\text { 1234123, 1234234, 1234312, 1234321, } \\
\text { 1234412, 1234421, 1234423, 1234431, } \\
1234432\end{array}$ & $\begin{array}{l}1233412 \sim 1233421,1234312 \sim 1234321 \text { and } 1234412 \sim \\
1234421 \text { by Corollary } 40,1231234 \sim 1233214 \text { by Corollary } 21 \text {, } \\
1231234 \sim 1234123 \text { by Theorem } 30,1234123 \sim 1234321 \text { by } \\
\text { Corollary } 20,1233421 \sim 1234321 \text { by Theorem } 42,1234321 \sim \\
1234421 \sim 1234431 \sim 1234432 \text { by Proposition } 15\end{array}$ \\
\hline $1234231,1234341,1234342$ & Corollary 13 \\
\hline 1233441,1233442 & Corollary 13 \\
\hline $1231244,1232144,1232344,1233244$ & Corollary 13 \\
\hline 1213453,1223453 & Corollary 13 \\
\hline 1213435,1223435 & Corollary 13 \\
\hline 1213456,1223456 & Corollary 13 \\
\hline 1213455,1223455 & Corollary 13 \\
\hline $1213445,1213454,1223445,1223454$ & Corollary 13 \\
\hline $1231145,1232245,1233345$ & Corollary 13 \\
\hline 1213345,1223345 & Corollary 13 \\
\hline 1213443,1223443 & Corollary 13 \\
\hline 1213434,1223434 & Corollary 13 \\
\hline $1234313,1234424,1233413$ & $\begin{array}{l}1233413 \sim 1234313 \text { by Theorem } 54,1234313 \sim 1234424 \text { by } \\
\text { Corollary } 13\end{array}$ \\
\hline 1234131,1234242 & Corollary 13 \\
\hline 1233134,1233143 & Theorem 54 \\
\hline 1234133,1234244 & Corollary 13 \\
\hline 1231334,1231433 & Theorem 54 \\
\hline 1234113,1234224 & Corollary 13 \\
\hline 1231242,1232142 & Theorem 54 \\
\hline 1232412,1232421 & Theorem 54 \\
\hline 1231124,1232234 & Corollary 13 \\
\hline $1234121,1234232,1234343$ & Corollary 13 \\
\hline 1231214,1232324 & Corollary 13 \\
\hline $\begin{array}{l}1231224,1232124,1232214,1232334, \\
1232343,1232433,1233234,1233243, \\
1233324,1233341,1233342,1233423, \\
1233431,1233432,1234112,1234122, \\
1234212,1234221,1234223,1234233, \\
1234323,1234331,1234332,1234334, \\
1234344,1234434,1234441,1234442, \\
1234443\end{array}$ & Corollary 13 \\
\hline 1213344,1223344 & Corollary 13 \\
\hline 1123445,1123454 & Theorem 41 \\
\hline 1232114,1233224 & Corollary 13 \\
\hline $\begin{array}{l}1233411,1233422,1234211,1234311, \\
1234322,1234411,1234422,1234433\end{array}$ & Corollary 13 \\
\hline $1231444,1232444,1233444$ & Corollary 13 \\
\hline $1231144,1232244,1233344$ & Corollary 13 \\
\hline $1212334,1212343,1221334,1221343$ & $\begin{array}{l}1212334 \sim 1221334 \text { and } 1212343 \sim 1221343 \text { by Corollary } 40, \\
1212334 \sim 1212343 \text { by Theorem } 41\end{array}$ \\
\hline 1212345,1221345 & Corollary 40 \\
\hline 1212344,1221344 & Corollary 40 \\
\hline
\end{tabular}


Table 5: Nonsingleton equivalence classes of patterns of size seven.

\begin{tabular}{|c|c|}
\hline Pattern & Reference \\
\hline $\begin{array}{l}1213334,1213343,1213433,1223334, \\
1223343,1223433\end{array}$ & $\begin{array}{l}1213334 \sim 1213343 \sim 1213433 \text { by Theorem } 41,1213334 \sim \\
1223334 \text { by Corollary } 13\end{array}$ \\
\hline 1213444,1223444 & Corollary 13 \\
\hline $\begin{array}{l}1231114,1232224,1233334,1233343, \\
1233433,1234111,1234222,1234333, \\
1234444\end{array}$ & Corollary 13 \\
\hline $1211334,1211343,1222334,1222343$ & Corollary 13 \\
\hline 1122334,1122343 & Theorem 41 \\
\hline 1211345,1222345 & Corollary 13 \\
\hline 1211344,1222344 & Corollary 13 \\
\hline $1123334,1123343,1123433$ & Theorem 41 \\
\hline $1212234,1221234,1222134$ & Corollary 40 \\
\hline$\overline{1233312,1233321}$ & Corollary 40 \\
\hline $1233122,1233212,1233221$ & Corollary 40 \\
\hline $1212233,1221233,1222133$ & Corollary 40 \\
\hline 1212333,1221333 & Corollary 40 \\
\hline 1121334,1121343 & Theorem 41 \\
\hline 1211134,1222234 & Corollary 13 \\
\hline 1112334,1112343 & Theorem 41 \\
\hline 1231212,1232323 & Corollary 13 \\
\hline 1232121,1233232 & Corollary 13 \\
\hline 1231221,1232332 & Corollary 13 \\
\hline 1232112,1233223 & Corollary 13 \\
\hline$\overline{1231122,1232233}$ & Corollary 13 \\
\hline $\begin{array}{l}\text { 1222311, 1223211, 1232211, 1233311, } \\
1233322\end{array}$ & $\begin{array}{l}1222311 \sim 1223211 \sim 1232211 \text { by Theorem } 42,1232211 \sim \\
1233311 \sim 1233322 \text { by Corollary } 13\end{array}$ \\
\hline 1211333,1222333 & Corollary 13 \\
\hline $\begin{array}{l}1212223,1212232,1212322,1213222, \\
\text { 1221223, 1221232, 1221322, 1222123, } \\
\text { 1222132, 1222213, 1222231, 1222312, } \\
\text { 1222321, 1223122, 1223212, 1223221, } \\
\text { 1231222, 1232122, 1232212, 1232221, } \\
\text { 1232333, 1233233, 1233323, 1233331, } \\
1233332\end{array}$ & $\begin{array}{l}1232221 \sim 1233331 \sim 1233332 \text { by Proposition } 15, \text { the rest by } \\
\text { Theorem } 48\end{array}$ \\
\hline 1231112,1232223 & Corollary 13 \\
\hline 1231121,1232232 & Corollary 13 \\
\hline 1231211,1232322 & Corollary 13 \\
\hline 1213333,1223333 & Corollary 13 \\
\hline $1223111,1232111,1233111,1233222$ & $\begin{array}{l}1223111 \sim 1232111 \text { by Theorem } 42,1232111 \sim 1233111 \sim \\
1233222 \text { by Proposition } 15\end{array}$ \\
\hline 1211133,1222233 & Corollary 13 \\
\hline $1122223,1122232,1122322,1123222$ & Theorem 41 \\
\hline $1112223,1112232,1112322$ & Theorem 41 \\
\hline $\begin{array}{l}1211113,1222223,1222232,1222322, \\
1223222,1231111,1232222,1233333\end{array}$ & Corollary 13 \\
\hline 1111223,1111232 & Theorem 41 \\
\hline $\begin{array}{l}1212222,1221222,1222122,1222212, \\
1222221\end{array}$ & Corollary 40 \\
\hline
\end{tabular}


Table 5: Nonsingleton equivalence classes of patterns of size seven.

\begin{tabular}{|l|l|}
\hline Pattern & Reference \\
\hline \hline $1111112,1111121,1111211,1112111$, & Theorems 34 and 36 \\
$1121111,1211111,1222222$ & \\
\hline
\end{tabular}

\section{Concluding remarks}

In Table 6, we present the total number of equivalence classes of patterns of length $1,2, \ldots, 7$.

\begin{tabular}{|c|c|c|c|c|c|c|c|}
\hline$n$ & 1 & 2 & 3 & 4 & 5 & 6 & 7 \\
\hline \hline number of classes of patterns of size $n$ & 1 & 1 & 2 & 5 & 21 & 114 & 617 \\
\hline
\end{tabular}

Table 6: Number of equivalence classes of patterns of size $1,2, \ldots, 7$

To provide an accurate asymptotic estimate of the number of equivalence classes of patterns of a given size remains out of reach of our methods.

Let us remark that our computer enumeration has revealed several pairs of nonequivalent patterns $\tau \not \tau^{\prime}$ whose growth functions $p(n ; \tau)$ and $p\left(n ; \tau^{\prime}\right)$ coincide for several small values of $n$. For instance, the growth functions of the two patterns $\tau=1234415$ and $\tau^{\prime}=1234152$ are equal for $n<15$. In other words, the value of $n=15$ is the smallest witness of the non-equivalence of the two patterns. We wonder if each two non-equivalent patterns of size $n$ have a witness of size $O(n)$, or even $n+O(1)$. Note that for any $k$ and for $\tau, \tau^{\prime}$ chosen as above, the pair of non-equivalent patterns $12 \cdots k(\tau+k)$ and $12 \cdots k\left(\tau^{\prime}+k\right)$ of length $k+7$ requires a witness of length $k+15$ (this follows from Theorem 31).

The raw enumeration data for patterns of size six and seven [27, 28] are available from the second author's website. To save space, we did not include the data in this paper. The data were obtained with the help of a computer program, whose source code is also available from the second author's website [29].

\section{References}

[1] A. V. Aho, R. Sethi, and J.D. Ullman, Compilers: principles, techniques and tools, Addison- Wesley, Reading, Mass., 1986

[2] J. Backelin, J. West, And G. XIN, Wilf-equivalence for singleton classes, Adv. Appl. Math. 32:2 (2007) 133-148.

[3] William Y. C. Chen, Eva Y.P. Deng, and Rosena R.X. Du, Reduction of m-regular noncrossing Partitions, Europ. J. Combin. 26:2 (2005) 237-243.

[4] William Y. C. Chen, Eva Y.P. Deng, Rosena R.X. Du, R. P. Stanley, and Catherine H. Yan, Crossings and Nestings of Matchings and Partitions, Trans. Amer. Math. Soc. 359(4) (2007) 1555-1575. 
[5] L. Comtet, Advanced Combinatorics, Presses Universitaires de France Io8, Boulevard Saint-Germain, Paris, 1970.

[6] H. Davenport And A. Schinzel, A combinatorial problem connected with differential equations, Amer. J. Math. 87 (1965) 684-694.

[7] A. DE MIER, $k$-noncrossing and $k$-nonnesting graphs and fillings of Ferrers diagrams, arXiv: math.CO/0602195.

[8] A. DE Mier, On the Symmetry of the Distribution of $k$-crossings and $k$-nestings in Graphs, Elect. J. Combin. 13 (2006) \#N21.

[9] P. Flajolet and R. Sedgewick Analytic Combinatorics, Part A, Chapter II; available at http://algo.inria.fr/ flajolet/Publications/books.html, 2006.

[10] W. Fulton, Young Tableaux, London Mathematical Society Student Texts 35; Cambridge University Press, 1997.

[11] A. M. GOYT, Avoidance of partitions of a three-element set, arXiv: math.CO/0603481.

[12] J.E. Hopcroft, R. Motwani, And J.D. Ullman, Introduction to automata theory, languages, and computation, Addison-Wesley, 2001

[13] M. Klazar, On abab-free and abba-free set partitions, Europ. J. Combin. 17 (1996) 53-68.

[14] M. Klazar, On trees and noncrossing partitions, Discr. Appl. Math. 82 (1998) $263-269$.

[15] C. Krattenthaler, Growth diagrams, and increasing and decreasing chains in fillings of Ferrers shapes, Adv. Appl. Math. 37:3 (2006) 404-431.

[16] G. Kreweras, Sur les partitions non croisées d'un cycle, Discrete Math. 1:4 (1972) $333-350$.

[17] T. Mansour, Combinatorial methods and recurrence relations with two indices, $J$. Diff. Eq. Appl. 12:6 (2006) 555-563.

[18] T. Mansour and S. Severini, Enumeration of $(k, 2)$-noncrossing partitions, Discrete Mathematics, to appear.

[19] R. C. Mullin And R. G. Stanton, A map-theoretic approach to DavenportSchinzel sequences, Pacific J. Math. 40 (1972) 167-172.

[20] M. Rubey, Increasing and decreasing sequences in fillings of moon polyominoes, arxiv: math.CO/0604140.

[21] B. SAgan, Pattern avoidance in set partitions, arxiv: math.C0/0604292.

[22] N. J. A. Sloane (ED.), The On-Line Encyclopedia of Integer Sequences (http://www.research.att.com/ njas/sequences/).

[23] Z. Stankova And J. West, A new class of Wilf-equivalent permutations, J. Alg. Combin. 15 (2002) 271-290.

[24] R. P. Stanley, Enumerative Combinatorics, Vol. 1, Cambridge University Press, Cambridge, UK, 1996. 
[25] R. P. Stanley, Enumerative Combinatorics, Vol. 2, Cambridge Studies in Advanced Mathematics 62, Cambridge University Press, 1999.

[26] M. Wachs and D. White, p,q-Stirling numbers and set partition statistics, $J$. Combin. Theory, Series A, 56:1 (1991) 27-46.

[27] http://math.haifa.ac.il/toufik/enumerative/tablepart6.html.

[28] http://math.haifa.ac.il/toufik/enumerative/tablepart7.html.

[29] http://math.haifa.ac.il/toufik/program/partitions.html. 